\title{
Invariant Borel liftings for category algebras of Baire groups
}

\author{
by
}

\author{
Maxim R. Burke (Charlottetown)
}

\begin{abstract}
R. A. Johnson showed that there is no translation-invariant Borel lifting for the measure algebra of $\mathbb{R} / \mathbb{Z}$ equipped with Haar measure, a result which was generalized by M. Talagrand to non-discrete locally compact abelian groups and by J. Kupka and K. Prikry to arbitrary non-discrete locally compact groups. In this paper we study analogs of these results for category algebras (the Borel $\sigma$-algebra modulo the ideal of first category sets) of topological groups. Our main results are for the class of non-discrete separable metric groups. We show that if $G$ in this class is weakly $\alpha$-favorable, then the category algebra of $G$ has no left-invariant Borel lifting. (This particular result does not require separability and implies a corresponding result for locally compact groups which are not necessarily metric.) Under the Continuum Hypothesis, many groups in the class have a dense Baire subgroup which has a left-invariant Borel lifting. On the other hand, there is a model in which the category algebra of a Baire group in the class never has a left-invariant Borel lifting. The model is a variation on one constructed by A. W. Miller and the author where every second category set of reals has a relatively second category intersection with a nowhere dense perfect set.
\end{abstract}

1. Introduction. A lifting for a structure $(X, \Sigma, \mathcal{N})$, where $X$ is a nonempty set, $\Sigma$ is a $\sigma$-algebra of subsets of $X$ and $\mathcal{N} \subseteq \Sigma$ is a $\sigma$-ideal of subsets of $X$, is a Boolean homomorphism $\Sigma / \mathcal{N} \rightarrow \Sigma$ which selects a representative from each equivalence class. Equivalently, a lifting is a Boolean homomorphism $\theta: \Sigma \rightarrow \Sigma$ such that $\theta(E) \triangle E \in \mathcal{N}$, and for all $E, F \in \Sigma$, $E \triangle F \in \mathcal{N}$ implies $\theta(E)=\theta(F)$. If $(X, \Sigma, \mu)$ is a complete $\sigma$-finite measure space and we take $\mathcal{N}$ to be the ideal of $\mu$-null sets, then $(X, \Sigma, \mathcal{N})$ has a lifting [Ma1958]. If $X$ is a locally compact group and $\mu$ is left Haar measure, then a lifting exists which commutes with left translations (i.e., $\theta(x E)=x \theta(E)$ ) [IT1967]. In the case of Lebesgue measure on the real line, if the Contin-

2000 Mathematics Subject Classification: Primary 54H11; Secondary 03E35, 03E50.

Key words and phrases: regular open algebra, property of Baire, invariant lifting, Cantor set, oracle chain condition forcing.

Research supported by NSERC. The author thanks the Department of Mathematics at the University of Toronto for its hospitality while this work was initiated. 
uum Hypothesis holds then the representatives chosen by a lifting can be taken to be Borel sets [vN] but it is also consistent that no such Borel lifting exists [Sh1983]. R. A. Johnson [Jo] proved that there is no Borel lifting for Haar measure on $\mathbb{R} / \mathbb{Z}$ which commutes with translations. This was subsequently generalized to non-discrete locally compact abelian groups $[\mathrm{Ta}]$ and then to arbitrary non-discrete locally compact groups [KP]. (The result in the non-abelian setting is that there is no Borel lifting for left Haar measure which commutes with left translations.) The method of [Ta] was adapted to arbitrary locally compact groups in [Lo] and [Bu1993a].

In this paper, we deal with liftings for $(X, \operatorname{BP}(X), \mathcal{M}(X))$ where $X$ is a topological space, $\mathrm{BP}(X)$ is the $\sigma$-algebra of sets $E$ which have the property of Baire, i.e., for which there is an open set $U$ such that $E \triangle U$ is first category, and $\mathcal{M}(X)$ is the collection of all first category (= meager) sets in $X$. When the space $X$ is clear from the context, we shall write $\mathrm{BP}$ and $\mathcal{M}$ instead of $\operatorname{BP}(X)$ and $\mathcal{M}(X)$, respectively. We shall also use the notation $E=\mathcal{M} F$ to mean $E \triangle F \in \mathcal{N}$. A lifting for $(X, \mathrm{BP}, \mathcal{M})$ is also called a lifting for the category algebra $\mathrm{BP} / \mathcal{M}$ of $X$.

It might as well be assumed that $X$ is a Baire space (i.e., no non-empty open set is first category) when we deal with $\mathrm{BP} / \mathcal{M}$, since, by a well-known result of Banach (see, e.g., [Kur, Theorem I.10.III.1]), the union of the open first category sets of $X$ is a first category set and its complement is then a Baire space which clearly has the same category algebra as $X$. A group which is not first category is automatically Baire by invariance of the topology. An important tool for working with Baire spaces is the following game characterization. A topological space $X$ is Baire if and only if Player I does not have a winning strategy in the Banach-Mazur game for two players, I and II, in which, starting with Player I, the players alternately play the terms of a decreasing sequence $U_{1} \supseteq U_{2} \supseteq \cdots$ of non-empty open sets and Player I wins if the intersection of the sequence is empty. (See [Re, Theorem 2.1].) When the stronger condition that Player II has a winning strategy holds, $X$ is called weakly $\alpha$-favorable.

Category analogs of some of the measure-theoretic results on liftings mentioned in the previous paragraphs have been considered in the literature. See, e.g., [LMZ]. In [Ma1977] it was observed in particular that a lifting for the category algebra of $X$ always exists. Liftings for product spaces which relate nicely to liftings for the factors were studied in [BMMS]. In the present paper, we establish category analogs of the above-mentioned results on translation-invariance and prove the consistency result that no non-discrete Baire separable metric group has a left-invariant Borel lifting for its category algebra. We do not know whether there is a measure-theoretic analog of this result. 
Problem 1.1. If $\lambda^{*}$ is Lebesgue outer measure on $\mathbb{R}, \mathcal{M}$ is the $\sigma$-algebra of Lebesgue measurable sets and $G$ is a subgroup of $\mathbb{R}$, equip $G$ with the $\sigma$-algebra $\mathcal{M}_{G}=\{M \cap G: M \in \mathcal{M}\}$ and the measure $\lambda^{*}$ restricted to $\mathcal{M}_{G}$. Is it consistent with ZFC that for no subgroup $G$ of $(\mathbb{R},+)$ does the measure algebra of $G$ have an invariant Borel lifting?

(Only subgroups of positive outer measure are of interest but it is not necessary to require $\lambda^{*} G>0$. Indeed, a structure $(X, \Sigma, \mathcal{N})$ has no lifting if $X \in \mathcal{N}$, since we need $\theta(\emptyset)=\emptyset$ and $\theta(X)=X$ but then $\emptyset$ and $X$ are equal modulo $\mathcal{N}$ whereas $\theta(\emptyset) \neq \theta(X)$, contradicting one of the requirements in the definition of a lifting.)

Our topological terminology follows [En]. For set theory and forcing terminology see [Kun]. For oracle-cc forcing, see [Sh1998]. We write $e$ for the identity element of an abstract group $G$. For each $i<\omega$, let $h_{i}$ : $2^{\omega} \rightarrow 2^{\omega}$ be the homeomorphism which interchanges 0 and 1 on the $i$ th coordinate, namely, $h_{i}(t)(j)=t(j)$ if $j \neq i$ and $h_{i}(t)(i)=1-t(i)$.

The author thanks W. Weiss and I. Farah for helpful discussions of this work and S. Solecki for telling him about Theorem 2.6.

2. Preliminary results. In this section, we gather results which are either known or whose proofs are not substantially different from those of known measure-theoretic analogs.

Unlike its counterpart for Haar measure mentioned in the previous section, the following result is easy to prove. The reason for this is that, unlike the situation for abstract measure spaces, there is always a natural choice of a representative for each category class, namely the regular open representative. Cf. [Ma1977, (1), p. 130].

Proposition 2.1. For any Baire topological group $G$, there is a leftinvariant lifting $\theta$ for $(G, \mathrm{BP}, \mathcal{M})$.

Proof. For each $E \in \mathrm{BP}$, let $d(E)$ be the unique regular open set in the class of $E$ in the category algebra. Note that $d$ preserves finite intersections and commutes with translations. Let $\mathcal{F}$ be an ultrafilter of subsets of $G$ extending the filter $\{E \in \mathrm{BP}: e \in d(E)\}$. Define

$$
\theta(E)=\left\{x \in G: x^{-1} E \in \mathcal{F}\right\} .
$$

That $\mathcal{F}$ is an ultrafilter easily implies that $\theta$ is a Boolean homomorphism. It is clear from the definition that $E=\mathcal{M} F$ implies $\theta(E)=\theta(F)$. For any $E \in \mathrm{BP}$, we have $d(E) \subseteq \theta(E) \subseteq d\left(E^{\mathrm{c}}\right)^{\mathrm{c}}$ so that $\theta(E)=\mathcal{M} d(E)=\mathcal{M} E$. For left-invariance we have $\theta(y E)=\left\{x \in G: x^{-1} y E \in \mathcal{F}\right\}=\{x \in G$ : $\left.\left(y^{-1} x\right)^{-1} E \in \mathcal{F}\right\}=y\left\{z \in G: z^{-1} E \in \mathcal{F}\right\}=y \theta(E)$. 
The following simple but important result follows by replacing measure by category in standard proofs of the measure-theoretic analog. For the reader's convenience we give a proof. Our presentation is taken from [Fr2003, 447B].

Proposition 2.2. Let $G$ be a Baire topological group, and let $\theta: \operatorname{BP}(G)$ $\rightarrow \mathrm{BP}(G)$ be a left-invariant lifting for the category algebra of $G$. Then $\theta$ is strong, i.e., $U \subseteq \theta(U)$ for each open set $U$.

Proof. Let $U$ be an open neighborhood of $e$. Let $V$ be an open neighborhood of $e$ such that $V^{-1} V \subseteq U$. Choose $v \in V \cap \theta(V)$. (Since $G$ is Baire, $V \notin \mathcal{M}(G)$.) Then $e=v^{-1} v \in v^{-1} \theta(V)=\theta\left(v^{-1} V\right) \subseteq \theta\left(V^{-1} V\right) \subseteq \theta(U)$. If $U$ is now any open set and $x \in U$, then $x^{-1} U$ is an open neighborhood of $e$ and hence $e \in \theta\left(x^{-1} U\right)$. This gives $x=x e \in x \theta\left(x^{-1} U\right)=\theta\left(x x^{-1} U\right)=\theta(U)$. Hence, $U \subseteq \theta(U)$.

The following lemma provides our framework for establishing the nonexistence of invariant Borel liftings. It is a version of the main idea of [Ta]. (Cf. the proof of [Lo, Theorem 1].)

Lemma 2.3. Let $G$ be a Hausdorff topological group and $H \subseteq G$ a dense Baire subgroup. Let $\left\langle m_{i}: i<\omega\right\rangle$ be a sequence of non-zero elements of $\omega$ and let

$$
\varphi:\left(\prod_{i<\omega} m_{i}\right) \times 2^{\omega} \rightarrow G
$$

be a homeomorphism onto a nowhere dense set $C$. Let $V \subseteq G$ be a Borel set. Suppose that the following conditions are satisfied:

(a) $C \cap H$ is a dense Baire subset of $C$.

(b) For each $(s, t) \in\left(\prod_{i<\omega} m_{i}\right) \times 2^{\omega}$ and each $i<\omega$, there is an open set $S \subseteq G$ containing $\varphi(s, t)$ and there is an $\alpha \in H$ such that $\varphi\left(s, h_{i}(t)\right)=\alpha \varphi(s, t)$ and $S \backslash C$ is partitioned by its intersections with $V$ and $\alpha^{-1} V$.

Then for any left-invariant lifting $\theta$ for $(H, \mathrm{BP}, \mathcal{M}), \theta(V \cap H) \cap C$ does not have the property of Baire relative to $C \cap H$. In particular, $\theta$ is not a Borel lifting.

In (b) it would be enough to assume the property when $\varphi(s, t) \in H$. Note that (b) implies that if $\varphi(s, t) \in H$ then for all $i<\omega, \varphi\left(s, h_{i}(t)\right) \in H$.

Proof. Fix $s \in \prod_{i<\omega} m_{i}$ and $t \in 2^{\omega}$ such that $\varphi(s, t) \in H$ and fix $i<\omega$. Let $S$ and $\alpha$ be as in (b). By Proposition 2.2 and because $C \cap H$ is nowhere dense in $H(C \cap H$ is nowhere dense in $G$ and $H$ is dense in $G)$, we see that $S \cap H \subseteq \theta(S \cap H)=\theta((S \backslash C) \cap H)$ is partitioned by its intersections with $\theta(V \cap H)$ and $\theta\left(\alpha^{-1} V \cap H\right)$. Since $\alpha \in H$ and $\theta$ is left-invariant, we have $\theta\left(\alpha^{-1} V \cap H\right)=\theta\left(\alpha^{-1}(V \cap H)\right)=\alpha^{-1} \theta(V \cap H)$. Hence, precisely one of 
$\varphi(s, t) \in \theta(V \cap H), \varphi\left(s, h_{i}(t)\right)=\alpha \varphi(s, t) \in \theta(V \cap H)$ holds. Thus, precisely one of $(s, t) \in \varphi^{-1}(\theta(V \cap H)),\left(s, h_{i}(t)\right) \in \varphi^{-1}(\theta(V \cap H))$ holds. It follows that $A=\varphi^{-1}(\theta(V \cap H))$ does not have the property of Baire relative to $Y=\varphi^{-1}(C \cap H)$. Indeed, if it did, then either $A$ or $Y \backslash A$ would contain a basic open set $([u] \times[v]) \cap Y$ modulo $\mathcal{M}(Y)$, say $A$ contains such a set. (For some $n<\omega, u \in \prod_{j<n} m_{j}$, and $v \in 2^{n}$, $[u] \times[v]$ denotes the set of all $(s, t) \in \operatorname{dom} \varphi$ such that $u \subseteq s$ and $v \subseteq t$.) Fix any $i<\omega$ such that $i \geq|v|$. Then the map $(s, t) \mapsto\left(s, h_{i}(t)\right)$ restricts to a homeomorphism of $[u] \times[v]$ which carries the trace of $A$ precisely to the trace of $Y \backslash A$. Since $A$ is residual in $([u] \times[v]) \cap Y$, it follows that so is $Y \backslash A$. But this is impossible since $([u] \times[v]) \cap Y$ is second category by (a).

The following proposition isolates a version for category of the argument from the first paragraph of the proof of [Bu1993a, Theorem 2.1].

Proposition 2.4. Let $G$ be a weakly $\alpha$-favorable topological group, let $K$ be a normal subgroup of $G$ such that $G / K$ is metrizable and let $\pi: G \rightarrow G / K$ be the projection map. Suppose that in $G / K$ there are a Borel set $U$ and a set $C$ such that $\pi^{-1}(C)$ is weakly $\alpha$-favorable and for any left-invariant lifting $\bar{\theta}$ for $(G / K, \mathrm{BP}, \mathcal{M}), \bar{\theta}(U) \cap C$ does not have the property of Baire relative to $C$. Then the sets $\pi^{-1}(U)$ and $\pi^{-1}(C)$ have the same properties in $G$, i.e., for any left-invariant lifting $\theta$ for $(G, \mathrm{BP}, \mathcal{M}), \theta\left(\pi^{-1}(U)\right) \cap \pi^{-1}(C)$ does not have the property of Baire relative to $\pi^{-1}(C)$.

Proof. We note for emphasis the elementary fact that since $K$ is normal, for any set $A \subseteq G$ we have $K A=\bigcup_{y \in A} K y=\bigcup_{y \in A} y K=A K$ and hence any left $K$-invariant set is $K$-invariant. Let $\theta$ be a left-invariant lifting for $(G, \mathrm{BP}, \mathcal{M})$. Define $\bar{\theta}: \mathrm{BP}(G / K) \rightarrow \mathrm{BP}(G / K)$ by the formula

$$
\bar{\theta}(E)=\pi\left(\theta\left(\pi^{-1}(E)\right)\right) .
$$

Because $\pi$ is open [HR, Theorem 5.17], the preimage under $\pi$ of each nowhere dense set is nowhere dense. Hence, the preimage of each meager set is meager. It follows that the preimage of each set with the property of Baire has the property of Baire. Hence $\theta\left(\pi^{-1}(E)\right)$ is defined for each $E \in \operatorname{BP}(G / K)$. Clearly, $\bar{\theta}$ preserves unions. Moreover, because $\pi^{-1}(E)$ is $K$-invariant and $\theta$ is left-invariant, it follows that $\theta\left(\pi^{-1}(E)\right)$ is $K$-invariant. Because $\theta\left(\pi^{-1}(E)\right)$ and $\theta\left(\pi^{-1}\left(E^{\mathrm{c}}\right)\right)$ are complementary $K$-invariant sets, $\pi$ maps them to complementary sets. Thus, $\bar{\theta}$ is a Boolean homomorphism. The image under $\pi$ of a $K$-invariant (i.e., full preimage) meager set is meager by Proposition 2.5. If $E \in \operatorname{BP}(G / K)$ and $U$ is the regular open set in the class of $\theta\left(\pi^{-1}(E)\right)$, then $U$ is $K$-invariant. Hence $M=U \triangle \theta\left(\pi^{-1}(E)\right)$ is $K$-invariant. We see that $\bar{\theta}(E)=\pi\left(\theta\left(\pi^{-1}(E)\right)\right)=\pi(U) \triangle \pi(M)$ has the property of Baire. If $E_{1} \triangle E_{2}$ is meager, then $\pi^{-1}\left(E_{1}\right) \triangle \pi^{-1}\left(E_{2}\right)=$ $\pi^{-1}\left(E_{1} \triangle E_{2}\right)$ is meager and hence $\theta\left(\pi^{-1}\left(E_{1}\right)\right)=\theta\left(\pi^{-1}\left(E_{2}\right)\right)$. Thus, $\bar{\theta}\left(E_{1}\right)=$ 
$\bar{\theta}\left(E_{2}\right)$. For open $U \subseteq G / K$, we have $\pi^{-1}(U) \subseteq \theta\left(\pi^{-1}(U)\right)$ since $\theta$ is strong. Applying $\pi$ gives $U \subseteq \bar{\theta}(U)$. Since the same is true for the interior of the complement of $U$, we get $\bar{\theta}(U)={ }_{\mathcal{M}} U$. Finally, if $g \in G$, then

$$
\begin{aligned}
\pi(g) \bar{\theta}(E) & =\pi(g) \pi\left(\theta\left(\pi^{-1}(E)\right)\right)=\pi\left(g \theta\left(\pi^{-1}(E)\right)\right)=\pi\left(\theta\left(g \pi^{-1}(E)\right)\right) \\
& =\pi\left(\theta\left(\pi^{-1}(\pi(g) E)\right)\right)=\bar{\theta}(\pi(g) E) .
\end{aligned}
$$

[For the second last equality, note that $x \in \pi^{-1}(\pi(g) E)$ gives $\pi(x) \in \pi(g) E$, $\pi\left(g^{-1} x\right) \in E$. Then we get $x=g\left(g^{-1} x\right) \in g \pi^{-1}(E)$.]

So $\bar{\theta}$ is a left-invariant lifting for the category algebra of $G / K$. Suppose $\theta\left(\pi^{-1}(U)\right) \cap \pi^{-1}(C)$ has the property of Baire relative to $\pi^{-1}(C)$. We then find that $\bar{\theta}(U) \cap C=\pi\left(\theta\left(\pi^{-1}(U)\right)\right) \cap C=\pi\left(\theta\left(\pi^{-1}(U)\right) \cap \pi^{-1}(C)\right)$ has the property of Baire relative to $C$ since $\pi^{-1}(C)$ is weakly $\alpha$-favorable and the restriction of $\pi$ to $\pi^{-1}(C)$ is an open map onto $C$. (See the proof that $\bar{\theta}(E)$ has the property of Baire.) This contradicts the properties of $U$ and $C$.

In [Bu1993a], a form of the Kuratowski-Ulam theorem for group quotients was established. The following proposition, without the assumption that $Y$ is metrizable (see [Bu1993a, Theorem 1.5]), was left as an exercise for the reader with the claim that it covered an easier special case of the Kuratowski-Ulam result which sufficed for the rest of that paper. Unfortunately, we do not see why this form of the statement is true. The special case where $Y$ is metrizable also suffices for the purposes of the main result of [Bu1993a] and for our purposes here. This time we give a careful proof of it.

Proposition 2.5. Let $X$ and $Y$ be topological spaces such that $X$ is weakly $\alpha$-favorable and $Y$ is metrizable. Let $\pi: X \rightarrow Y$ be an open continuous surjection. If $A \subseteq Y$ is second category, then $\pi^{-1}(A)$ is second category in $X$.

Proof. Note that for any topological space $T$ and any dense subspace $S, S$ is Baire if and only if the Banach-Mazur game in $T$, modified so that Player I wins if the intersection of the plays does not meet $S$, has no winning strategy for Player I. We denote this modified game by $\mathcal{G}(T, S)$.

By replacing $Y$ by a non-empty open subset $U$ in which $A$ is everywhere second category, and replacing $X$ by $\pi^{-1}(U)$, we may assume that $A$ is everywhere second category in $Y$. Then $\pi^{-1}(A)$ is dense in $X$ since $\pi$ is open. Let $\tau_{X}$ be a winning strategy for Player II in the Banach-Mazur game for $X$. Let $\sigma_{X}$ be a strategy for Player I in $\mathcal{G}\left(X, \pi^{-1}(A)\right)$. We must show that $\sigma_{X}$ is not winning. We use $\sigma_{X}$ and $\tau_{X}$ to describe a strategy $\sigma_{Y}$ for Player I in $\mathcal{G}(Y, A)$. We need to define $\sigma_{Y}\left(G_{0}, H_{0}, \ldots, G_{n}, H_{n}\right)$ corresponding to each play

$$
G_{0} \supseteq H_{0} \supseteq G_{1} \supseteq H_{1} \supseteq \cdots
$$

of the game $\mathcal{G}(Y, A)$. Only plays for which $G_{n}=\sigma_{Y}\left(G_{0}, H_{0}, \ldots, G_{n-1}, H_{n-1}\right)$ 
for each $n<\omega$ are relevant, so we limit ourselves to those. The definition involves defining an auxiliary sequence of open sets in $X$ :

$$
U_{0} \supseteq V_{0} \supseteq U_{1} \supseteq V_{1} \supseteq \cdots
$$

The definitions are as follows. For each non-empty open subset $G$ of $Y$ and each $n<\omega$, we fix a non-empty open $L(G, n) \subseteq G$ having diameter at most $2^{-n}$ in some fixed metric for $Y$. Now we let

(i) $U_{n}=\sigma_{X}\left(U_{0}, V_{0} \cap \pi^{-1}\left(H_{0}\right), \ldots, U_{n-1}, V_{n-1} \cap \pi^{-1}\left(H_{n-1}\right)\right)$,

(ii) $V_{n}=\tau_{X}\left(U_{0}, V_{0}, \ldots, U_{n-1}, V_{n-1}, U_{n}\right)$,

(iii) $G_{n}=\sigma_{Y}\left(G_{0}, H_{0}, \ldots, G_{n-1}, H_{n-1}\right)=L\left(\pi\left(V_{n}\right), n\right)$.

We check inductively that

(iv) $U_{0} \supseteq V_{0} \supseteq \cdots U_{n} \supseteq V_{n} \neq \emptyset$,

(v) $G_{0} \supseteq H_{0} \supseteq \cdots \supseteq G_{n} \supseteq H_{n} \neq \emptyset$,

(vi) $U_{0} \supseteq V_{0} \cap \pi^{-1}\left(H_{0}\right) \supseteq \cdots \supseteq U_{n} \supseteq V_{n} \cap \pi^{-1}\left(H_{n}\right) \neq \emptyset$.

Consider first what happens when $n=0$. Item (i) shows that $U_{0}=\sigma_{X}(\emptyset)$ is a non-empty open set. Then (ii) gives $V_{0} \subseteq U_{0}$ and $V_{0} \neq \emptyset$. Item (iii) then implies that $G_{0}=\sigma_{Y}(\emptyset)=L\left(\pi\left(V_{0}\right), 0\right)$ is a non-empty open set. Then by assumption $H_{0} \subseteq G_{0}$ and $H_{0}$ is non-empty. The inclusion in (vi) is a consequence of the inclusion in (iv).

Suppose that for some $n$ we have verified (iv) and (v) and all of (vi) except the inequality at the end. By (iii), $H_{n} \subseteq G_{n} \subseteq \pi\left(V_{n}\right)$ so that $V_{n} \cap$ $\pi^{-1}\left(H_{n}\right) \neq \emptyset$. Then (i) makes sense for $n+1$ and gives $U_{n+1} \subseteq V_{n}$. This, together with (iv), justifies (ii) for $n+1$ and gives $V_{n+1} \subseteq U_{n+1}$. Item (i) also gives $\pi\left(U_{n+1}\right) \subseteq H_{n}$. By (iii) for $n+1$ (which makes sense by (v)), $G_{n+1} \subseteq \pi\left(V_{n+1}\right) \subseteq \pi\left(U_{n+1}\right) \subseteq H_{n}$. Thus, (v) holds for $n+1$. The second last inclusion in (vi) for $n+1$ is immediate from the definition of $U_{n+1}$ as given by (i).

Since $A$ is Baire, the strategy $\sigma_{Y}$ is not winning in $\mathcal{G}(Y, A)$. Choose the responses $H_{n}$ for Player II so that $\bigcap_{n} G_{n}=\bigcap_{n} H_{n}$ meets $A$. By (iii), the intersection cannot contain more than one point. Hence there is a $y \in A$ such that $\bigcap_{n} G_{n}=\bigcap_{n} H_{n}=\{y\}$. Since $\tau_{X}$ is a winning strategy, there is a point $x \in \bigcap_{n} U_{n}=\bigcap_{n} V_{n}$. By (i), $\pi(x) \in H_{n}$ for all $n<\omega$. Hence $\pi(x)=y$, which gives $x \in \pi^{-1}(A)$. This shows that $\sigma_{X}$ is not winning for $\mathcal{G}\left(X, \pi^{-1}(A)\right)$ and hence that $\pi^{-1}(A)$ is Baire and hence everywhere second category in $X$.

We shall need the following fact about metrizable groups.

THEOREM 2.6 ([TH-J, Theorem 2.3.5, p. 352]). If $G$ is a metrizable topological group, then the topology of $G$ is generated by a left-invariant metric $d$, i.e., a metric satisfying $d(x, y)=d(z x, z y)$ for all $x, y, z \in G$. Moreover, the metric $\varrho$ given by

$$
\varrho(x, y)=d(x, y)+d\left(x^{-1}, y^{-1}\right)
$$


generates the topology of $G$ and, if $(\widetilde{G}, \widetilde{\varrho})$ denotes the completion of the metric space $(G, \varrho)$, then the group multiplication extends uniquely to $\widetilde{G}$ in such a way that $(\widetilde{G}, \widetilde{\varrho})$ is a topological group.

Notice that if we are given transitive models of set theory $M_{1} \subseteq M_{2}$ and a separable metric group $(G, d) \in M_{1}$, where $d$ is a left-invariant metric, then Theorem 2.6 gives natural embeddings of $(G, \varrho)$ into complete metric groups $\left(\widetilde{G}_{i}, \widetilde{\varrho}_{i}\right) \in M_{i}, i=1,2$, and in $M_{2}, \widetilde{G}_{2}$ can be considered in a natural way to be an extension of $\widetilde{G}_{1}$. Furthermore, in $M_{1}$, if we fix a countable dense set $G^{\prime} \subseteq G$ then the Borel subsets of $\widetilde{G}_{i}$ (and in fact we care only about the open sets and the closed sets) have natural countable codes describing how they are built using balls $\left\{x \in \widetilde{G}_{i}: \varrho(x, y)<\varepsilon\right\}$, where $y \in G^{\prime}$ and $\varepsilon$ is a positive rational number. In such circumstances, we shall simply write $\widetilde{G}$ instead of $\widetilde{G}_{1}$ or $\widetilde{G}_{2}$, leaving it to the context to indicate which group is intended.

We shall make use of the following well-known facts. We do not know a convenient reference for them in precisely the form we require, so we give the brief proofs. For a reference which essentially contains this, see $[\mathrm{Hu}$, Chapter V].

Proposition 2.7. Let $G$ be a topological group.

(a) Let $H$ be a subgroup of $G$. If for some $p \in H$ and some open neighborhood $U$ of $p$ the set $U \cap H$ is first category in $G$, then $H$ is first category in $G$.

(b) If $G$ is Baire and $A \subseteq G$ contains a residual subset of a non-empty open set, then $A A^{-1}$ is a neighborhood of the identity.

(c) (Open Mapping Theorem) Let $G$ and $H$ be Polish groups and let $f: G \rightarrow H$ be a continuous homomorphism. If the range of $f$ is second category, then $f$ is open.

Proof. (a) Let $q \in H$. The map $\phi: G \rightarrow G$ defined by $\phi(x)=q p^{-1} x$ is a homeomorphism of $G$ such that $\phi(U)$ is an open neighborhood of $\phi(p)=q$ and $\phi(U) \cap H=\phi(U) \cap \phi(H)=\phi(U \cap H)$ is a first category set. This shows that $H$ is locally first category at every one of its points and hence is first category by the result of Banach cited in the introduction ([Kur, Theorem I.10.III.1]).

(b) We may take the open set in question to have the form $U x$ for some $x \in G$ and some open neighborhood $U$ of the identity $e$. So our assumption is that $A \cap(U x)$ is residual in $U x$. Choose an open neighborhood $W$ of $e$ such that $W W \subseteq U$. For each $w \in W$, the set $(w A) \cap A$ contains a residual subset of $(w U x) \cap(U x)$ and the latter is non-empty since it contains $w w x$. Hence $(w A) \cap A$ is not empty, from which it follows that $w \in A A^{-1}$. Thus, $W \subseteq A A^{-1}$. 
(c) Let $V_{0}$ be an open neighborhood of the identity $e \in G$. Choose an open neighborhood $V$ of $e$ so that $V V^{-1} \subseteq V_{0}$. Since countably many translates of $V$ cover $G$, countably many translates of $f(V)$ cover $f(G)$. Since one of these must be second category in $H, f(V)$ is second category in $H$. Since $f$ is continuous and $V$ is open, $f(V)$ is analytic and hence has the property of Baire. By (b), $f(V) f(V)^{-1}=f\left(V V^{-1}\right) \subseteq f\left(V_{0}\right)$ is a neighborhood of the identity in $H$.

\section{Weakly $\alpha$-favorable metric groups}

THEOREM 3.1. Let $G$ be a weakly $\alpha$-favorable metrizable non-discrete group. Then there are an open set $U \subseteq G$ and a nowhere dense Cantor set $C \subseteq G$ such that for any left-invariant lifting $\theta$ for $(G, \operatorname{BP}(G), \mathcal{M}(G))$, $\theta(U) \cap C$ does not have the property of Baire relative to $C$.

Proof. Fix a winning strategy $\sigma$ for Player II in the Banach-Mazur game on $G$. By induction on $n<\omega$, we define $\alpha_{n} \in G$, and for $t \in 2^{n}$, we define, by induction of the lexicographic order on $2^{n}$, non-empty open sets $U_{t}^{n}$ and $W_{t}^{n}$ so that the following conditions are satisfied. (The mention of $n$ in this notation is redundant since $n=|t|$, but for the case where $t$ is the constant zero sequence, which we denote simply by 0 regardless of the value of $n$, the notation $U_{0}^{n}, W_{0}^{n}$ avoids ambiguity.)

(i) $U_{0}^{0}=G$.

(ii) For each $n<\omega, t \in 2^{n}$ and $i<2, U_{t}^{n} \supseteq W_{t}^{n} \supseteq U_{t i}^{n+1}$ and $U_{t 0}^{n+1} \cap$ $U_{t 1}^{n+1}=\emptyset$. The union $U_{t 0}^{n+1} \cup U_{t 1}^{n+1}$ is not dense in $W_{t}^{n}$.

(iii) $U_{t}^{n}=\beta_{t}^{n} U_{0}^{n}$, where

$$
\beta_{t}^{n}=\alpha_{0}^{t(0)} \cdots \alpha_{n-1}^{t(n-1)}
$$

(Take $\left.\beta_{0}^{0}=e.\right)$

(iv) For each $t \in 2^{n}$, we have

$$
\begin{aligned}
U_{0}^{0} \supseteq W_{0}^{0} & \supseteq \cdots \supseteq W_{t \uparrow k}^{k} \\
& \stackrel{(1)}{\supseteq} \beta_{t \uparrow(k+1)}^{k+1}\left(\beta_{s^{k+1}(t \uparrow(k+1))}^{k+1}\right)^{-1} W_{s^{k+1}(t \uparrow(k+1))}^{k+1} \stackrel{(2)}{\supseteq} W_{t \uparrow(k+1)}^{k+1} \supseteq \cdots \\
& \supseteq \beta_{t}^{n}\left(\beta_{s^{n}(t)}^{n}\right)^{-1} W_{s^{n}(t)}^{n},
\end{aligned}
$$

where for each $t \in 2^{n}, s^{n}(t)$ denotes the immediate predecessor of $t$ in the lexicographic order on $2^{n}$. Even though $s^{n}(0)$ does not exist, we set $W_{s^{n}(0)}^{n}=U_{0}^{n}, \beta_{s^{n}(0)}^{n}=e$. The sequence of inclusions stops at $W_{0}^{0}$ when $n=0$, the last inclusion being the special case of (1) where $k=n-1$ and hence applying only when $n>0$. The set $W_{t}^{n}$ is Player II's next move according to $\sigma$ when the sequence of plays so far has been as above. 
(v) $U_{0}^{n+1} \subseteq G$ is a non-empty open set, $\alpha_{n} \in G$ and $U_{0}^{n+1}$ and $\alpha_{n} U_{0}^{n+1}$ are disjoint subsets of $\left(\beta_{1}^{n}\right)^{-1} W_{1}^{n}$ whose union is not dense in $\left(\beta_{1}^{n}\right)^{-1} W_{1}^{n}$, where the subscript 1 denotes the constant sequence with value 1 in $2^{n}$. Furthermore, each of the sets $U_{0}^{n+1}$ and $\alpha_{n} U_{0}^{n+1}$ has diameter at most $1 /(n+1)$ in some fixed left-invariant metric for $G$.

To carry out the induction, start by defining $U_{0}^{0}=G$ and let $W_{0}^{0}$ be Player II's first move according to $\sigma$ in the game where Player I's first move is $U_{0}^{0}$. This takes care of (i), (iii), (iv) and the first inclusion in (ii) when $n=0$. Given $U_{t}^{n}$ and $W_{t}^{n}$ for $t \in 2^{n}$, define $U_{0}^{n+1}$ and $\alpha_{n}$ so that (v) holds. Since $G$ is not discrete, and hence has no isolated points, this is not a problem. Define other $U_{t}^{n+1}$ by the formula in (iii).

For the first part of (iv), fix $t \in 2^{n+1}$ and notice that, by the induction hypothesis, according to the first inclusion in (ii) and clause (iii), for $k+1 \leq n$ we have

$$
\begin{aligned}
\beta_{t \uparrow(k+1)}^{k+1}\left(\beta_{s^{k+1}(t\lceil(k+1))}^{k+1}\right)^{-1} W_{s^{k+1}(t \uparrow(k+1))}^{k+1} & \\
& \subseteq \beta_{t \uparrow(k+1)}^{k+1}\left(\beta_{s^{k+1}(t \uparrow(k+1))}^{k+1}\right)^{-1} U_{s^{k+1}(t \uparrow(k+1))}^{k+1} \\
& =\beta_{t \uparrow(k+1)}^{k+1} U_{0}^{k+1}=U_{t \uparrow(k+1)}^{k+1},
\end{aligned}
$$

and the last term, according to the second inclusion in (ii), is contained in $W_{t \uparrow k}^{k}$ so that the inclusions (1) hold. (Check separately the case where $t \uparrow(k+1)$ is the zero sequence.) The inclusions (2), for $k+1<n$, hold by the last part of (iv). Now define $W_{t}^{n+1}$ as in the second part of (iv). The first inclusion in (ii) (i.e., $W_{t}^{n+1} \subseteq U_{t}^{n+1}$ ) holds by the definition of $W_{t}^{n+1}$ and the calculation above with $k=n$, or directly from the definition of $W_{t}^{n+1}$ if $t=0$.

There remains to check the parts of clause (ii) (for $n$ ) with superscript $n+1$. For the inclusion, we have

$$
U_{t i}^{n+1}=\beta_{t i}^{n+1} U_{0}^{n+1}=\beta_{t}^{n} \gamma U_{0}^{n+1},
$$

where $\gamma=e$ or $\gamma=\alpha_{n}$ depending on whether $i=0$ or $i=1$. Either way, from (v) we deduce that

(*) $\quad U_{t i}^{n+1} \subseteq \beta_{t}^{n}\left(\beta_{1}^{n}\right)^{-1} W_{1}^{n}$ and $U_{t 0}^{n+1} \cup U_{t 1}^{n+1}$ is not dense in $\beta_{t}^{n}\left(\beta_{1}^{n}\right)^{-1} W_{1}^{n}$. The inclusion $W_{t}^{n} \subseteq \beta_{t}^{n}\left(\beta_{s^{n}(t)}^{n}\right)^{-1} W_{s^{n}(t)}^{n}$ from the last part of (iv) can be rewritten as

$$
\left(\beta_{t}^{n}\right)^{-1} W_{t}^{n} \subseteq\left(\beta_{s^{n}(t)}^{n}\right)^{-1} W_{s^{n}(t)}^{n} .
$$

Iterating this gives

$$
\left(\beta_{t}^{n}\right)^{-1} W_{t}^{n} \subseteq\left(\beta_{s}^{n}\right)^{-1} W_{s}^{n}
$$


whenever $s, t \in 2^{n}$ and $s$ precedes $t$ lexicographically. In particular, (**) holds for all $s$ when $t=1$. From $(*)$ we now get

$$
U_{t i}^{n+1} \subseteq \beta_{t}^{n}\left(\beta_{1}^{n}\right)^{-1} W_{1}^{n} \subseteq \beta_{t}^{n}\left(\beta_{t}^{n}\right)^{-1} W_{t}^{n}=W_{t}^{n}
$$

together with the last statement of (ii). Finally, $U_{t 0}^{n+1} \cap U_{t 1}^{n+1}=\left(\beta_{t}^{n} U_{0}^{n+1}\right) \cap$ $\beta_{t}^{n} \alpha_{n} U_{0}^{n+1}=\beta_{t}^{n}\left(U_{0}^{n+1} \cap \alpha_{n} U_{0}^{n+1}\right)=\emptyset$ by $(\mathrm{v})$.

By (ii) we have, for each $t \in 2^{\omega}$,

$$
U_{0}^{0} \supseteq W_{0}^{0} \supseteq \cdots \supseteq W_{t \uparrow k}^{k} \supseteq U_{t \uparrow(k+1)}^{k+1} \supseteq W_{t \uparrow(k+1)}^{k+1} \supseteq \cdots .
$$

Hence, $\bigcap_{n<\omega} U_{t \uparrow n}^{n}=\bigcap_{n<\omega} W_{t \uparrow n}^{n}$ and by (iv) the right-hand side equals the intersection of a sequence of plays of the Banach-Mazur game in which Player II uses a winning strategy. Hence, this intersection in non-empty. By (v), we have

$$
\bigcap_{n<\omega} U_{t\lceil n}^{n}=\{\varphi(t)\}
$$

for some $\varphi: 2^{\omega} \rightarrow G$. From (ii) and the last statement of (v), it follows that $\varphi$ is continuous and one-to-one. Let $C$ be the range of $\varphi$. By (ii) and (v), $C$ is nowhere dense in $G$. (If $\varphi(t)$ were in the interior, then by (v), $U_{t \uparrow n}^{n}$ is in the interior for some $n$. This contradicts (ii).) It also follows from (ii) that

$$
C=\bigcap_{n<\omega} \bigcup_{s \in 2^{n}} U_{s}^{n}
$$

The rest of the proof follows the argument for the 2ème étape of the proof in [Ta] (see also the proof of [Lo, Theorem 1]). For completeness, we reproduce the argument. For $x \in G \backslash C$, there is a largest $n=n(x)<\omega$ such that $x \in U_{t}^{n}$ for some (unique) $t=t(x) \in 2^{n}$. Define

$$
V=\left\{x \in G \backslash C: t_{0}+\cdots+t_{n-1} \text { is odd }\right\} .
$$

Then $V$ is the union of countably many sets of the form $U_{t}^{n} \backslash\left(U_{t 0}^{n+1} \cup U_{t 1}^{n+1}\right)$ and hence is Borel. If $i<n<\omega$ and $t \in 2^{\omega}$ satisfies $t(i)=0$, then by (iii) we have

$$
U_{h_{i}(t)\lceil n}^{n}=\beta_{t \uparrow i}^{i} \alpha_{i}\left(\beta_{t \uparrow i}^{i}\right)^{-1} U_{t\lceil n}^{n} .
$$

Similarly, if $t(i)=1$, then

$$
U_{h_{i}(t)\lceil n}^{n}=\beta_{t \uparrow i}^{i} \alpha_{i}^{-1}\left(\beta_{t \uparrow i}^{i}\right)^{-1} U_{t \uparrow n}^{n} .
$$

Claim. For any $t \in 2^{\omega}$ and any $i<\omega$, there is an open neighborhood $S$ of $\varphi(t)$ and there is an $\alpha \in G$ such that $\varphi\left(h_{i}(t)\right)=\alpha \varphi(t)$. Furthermore, $S \backslash C$ is partitioned by its intersections with $V$ and $\alpha^{-1} V$.

Proof. We can take for $S$ any $U_{t \mid m}^{m}$ with $i<m$. Intersecting over $n$ in the two displayed equations above shows that $\varphi\left(h_{i}(t)\right)=\alpha \varphi(t)$ where $\alpha=\beta_{t \uparrow i}^{i} \alpha_{i}^{ \pm 1}\left(\beta_{t \uparrow i}^{i}\right)^{-1}$. Now consider $x \in S \backslash C=U_{t \uparrow m}^{m} \backslash C$. We want to show that precisely one of $x, \alpha x$ belongs to $V$. For the unique $n$ such that 
$x \in U_{s}^{n} \backslash\left(U_{s 0}^{n} \cup U_{s 1}^{n}\right)$ for some $s \in 2^{n}$, we have $n \geq m$ since $x \in U_{t \uparrow m}^{m}$. Thus $i<n$ and we have

$$
\alpha x \in \alpha U_{s}^{n}=U_{h_{i}^{n}(s)}^{n} \quad \text { and } \quad \alpha x \notin \alpha U_{s j}^{n}=U_{h_{i}^{n}(s) j}^{n}, \quad j=0,1,
$$

where $h_{i}^{n}: 2^{n} \rightarrow 2^{n}$ interchanges 0 and 1 on the $i$ th coordinate. It follows that $\alpha x \notin C$ and the same unique $n$ witnesses this. The unique $r$ such that $x \in U_{r}^{n}$ is $r=s$ and the unique $r$ such that $\alpha x \in U_{r}^{n}$ is $r=h_{i}^{n}(s)$. Thus, precisely one of $x, \alpha x$ is in $V$. This gives the second statement of the Claim.

The proof is now completed using Lemma 2.3. The $U$ in the statement of the theorem is any open representative of the class of $V$ in the category algebra.

THEOREM 3.2. Let $G$ be a non-discrete locally compact Hausdorff group. Then there are an open set $U \subseteq G$ and a nowhere dense compact set $C$ such that for any left-invariant lifting $\theta$ for $(G, \operatorname{BP}(G), \mathcal{M}(G)), \theta(U) \cap C$ does not have the property of Baire relative to $C$.

Proof. As in the proof of [KP, Theorem 3.2], we have a compact normal subgroup $K$ of $G$ such that $G / K$ is second countable and not discrete. By [HR, Theorem 5.22], $G / K$ is locally compact. Hence Theorem 3.1 applies. Get $U$ and $C$ as given there for $G / K$. Let $\pi: G \rightarrow G / K$ be the projection map. Then $\pi^{-1}(C)$ is compact since $C$ is compact and $\pi$ is perfect. ( $\pi$ is closed since $G / K$ has the quotient topology and for any closed set $C \subseteq G, \pi^{-1}(\pi(C))=C K$ is closed.) Furthermore, $\pi^{-1}(C)$ is nowhere dense since $\pi$ is open. The rest follows from Proposition 2.4.

The next two results show that, assuming the Continuum Hypothesis $(\mathrm{CH})$, we cannot replace weakly $\alpha$-favorable by Baire in Theorem 3.1.

Proposition 3.3. Let $G$ be a Baire topological group. Let $L$ be a dense subgroup of $G$ which is a Luzin set, i.e., is uncountable but has countable intersection with each first category set. Then $L$ has a left-invariant Borel lifting for its category algebra.

Note that a subgroup which is a Luzin set is automatically a Baire space. This follows from Proposition 2.7(a).

Proof of Proposition 3.3. Take any left-invariant lifting $\theta$ for the category algebra of $G$. (There is such a lifting by Proposition 2.1.) Restricting to $L$ gives a left-invariant lifting for the category algebra of $L$. Indeed, for regular open $V \subseteq L$, there is a unique regular open $U \subseteq G$ such that $V=U \cap L$. Note that for $l \in L, l V=(l U) \cap L$. Define $\bar{\theta}(V)=\theta(U) \cap L$. Then $\bar{\theta}$ is a Boolean homomorphism and a selector for the category classes, and for $l \in L$,

$$
\bar{\theta}(l V)=\theta(l U) \cap L=(l \theta(U)) \cap L=l(\theta(U) \cap L)=l \bar{\theta}(V) .
$$


Now consider any regular open set $V \subseteq L$. We observe that $\bar{\theta}(V) \triangle V$ is first category and hence countable. Thus, $\bar{\theta}(V)$ is Borel.

Proposition 3.4. (CH) Let $G$ be a non-discrete Baire separable metric group. For positive integers $k, l$ and words $w\left(x_{1}, \ldots, x_{k}\right)$ on the alphabet $\left\{x_{1}, \ldots, x_{k}, a_{1}, \ldots, a_{l}\right\}$, where $x_{1}, \ldots, x_{k}$ are variables and $a_{1}, \ldots, a_{l} \in G$, let us call functions $G^{k} \rightarrow G, x \mapsto w(x)$, word maps.

(a) Suppose that each word map either has countable range or is open. Then there is a dense subgroup of $G$ which is a Luzin set.

(b) Suppose that some word map $G^{k} \rightarrow G, x \mapsto w(x)$, where $w$ is a word on the alphabet $\left\{x_{1}, \ldots, x_{k}\right\}$ (no constants), has first category range and nowhere dense fibers. Then $G$ has no subgroups which are Luzin sets.

Proof. (a) Let $\mathcal{B}$ be a countable base for the topology of $G$. Let $L \subseteq G$ be a set such that $L \cap B$ is second category for each non-empty $B \in \mathcal{B}$, and for each positive integer $n$, there do not exist uncountably many pairwise disjoint $n$-element subsets of $L$ which, when identified (arbitrarily) with some enumeration, form a nowhere dense subset of $G^{n}$. [See [To, Proposition 6.0] for the existence of such an $L$. The context there is the real line, but the same argument works for any Baire separable metric space. Briefly, by $\mathrm{CH}$, there is an increasing chain $\left\langle M_{\xi}: \xi<\omega_{1}\right\rangle$ of countable elementary submodels of $H_{\lambda}$, for a suitably large regular cardinal $\lambda$, such that $G \in M_{0}$ and $\mathbb{R} \subseteq \bigcup_{\xi<\omega_{1}} M_{\xi}$. (Note that then we also have $G \subseteq \bigcup_{\xi<\omega_{1}} M_{\xi}$ since $M_{0}$ contains a one-to-one function $G \rightarrow \mathbb{R}$.) Let $s: \omega_{1} \rightarrow \mathcal{B} \backslash\{\emptyset\}$ be such that $s^{-1}(B)$ is uncountable for each $B \in \mathcal{B} \backslash\{\emptyset\}$. Inductively choose $x_{\xi}, \xi<\omega_{1}$, so that for some model $M_{\eta(\xi)}$ containing $\left\{x_{\xi^{\prime}}: \xi^{\prime}<\xi\right\}, x_{\xi}$ is a member of $s(\xi)$ which is a Cohen real over $M_{\eta(\xi)}$. Then $L=\left\{x_{\xi}: \xi<\omega_{1}\right\}$ is as desired because if $N$ is a (code for a) closed nowhere dense subset of $G^{n}$, then, since $\mathbb{R} \subseteq \bigcup_{\xi<\omega_{1}} M_{\xi}$, we have $N \in M_{\eta}$ for some $\eta<\omega_{1}$. Then no $n$-tuple of distinct elements of $\left\{x_{\xi}: \eta<\xi<\omega_{1}\right\}$ belongs to $N$.]

We now verify that the subgroup of $G$ generated by $L$ is a Luzin set. If uncountably many words $w_{\zeta}, \zeta<\omega_{1}$, on the elements of $L$ belong to a closed nowhere dense set $N \subseteq G$, then we may assume that the subsets of $L$ involved all have the same cardinality $m$ and form a $\Delta$-system. We may enumerate them as $\left\{x_{0}, \ldots, x_{r-1}, x_{r}^{\zeta}, \ldots, x_{m-1}^{\zeta}\right\}$ where $\left\{x_{0}, \ldots, x_{r-1}\right\}$ enumerates the root and is the same for all words. We may also assume that there is a function $f: G^{m-r} \rightarrow G$ such that $f\left(y_{r}, \ldots, y_{m-1}\right)$ is given by a fixed word on the alphabet $\left\{x_{0}, \ldots, x_{r-1}\right\} \cup\left\{y_{r}, \ldots, y_{m-1}\right\}$, and for each $\zeta<\omega_{1}, w_{\zeta}=f\left(x_{r}^{\zeta}, \ldots, x_{m-1}^{\zeta}\right)$. Since the $w^{\zeta}$ are distinct, $f$ does not have countable range. Hence, $f$ is an open continuous map, so $f^{-1}(N)$ is a closed nowhere dense set in $G^{m-r}$. Since $f^{-1}(N)$ contains uncountably many disjoint $(m-r)$-tuples of $L$, we have a contradiction. 
(b) Let $f$ be the given word map. Suppose some subgroup $L$ were a Luzin set. Since the fibers of $f$ are nowhere dense, $L$ has a countable intersection with each one. Since $L$ itself is not countable, it follows that its image $f(L)$ is uncountable. The fact that $w$ contains no constants gives $f(L) \subseteq L$. By assumption, $f(L)$ is first category in $G$ and hence $L$ is not a Luzin set, a contradiction.

We can be more precise about the conditions under which there exist subgroups which are Luzin sets in abelian Polish groups.

Corollary 3.5. Let $G$ be a non-discrete abelian Polish group. There is a (dense) subgroup $L$ of $G$ which is a Luzin set if and only if, for each integer a, the map $x \mapsto$ ax has either an open range or an open kernel.

The parentheses around the word "dense" indicate that the statement is true with or without it.

Proof. Suppose the condition fails and $a$ is a counterexample. Since $x \mapsto a x$ is a continuous homomorphism, by the Open Mapping Theorem (Proposition 2.7(c)) the fact that the image is not open implies that the image is first category. Since the kernel of this map is a subgroup, the fact that it is not open implies that its interior is empty. Now apply Proposition 3.4(b).

Conversely, if the condition holds, we show that the hypothesis of Proposition 3.4(a) is satisfied. Since $G$ is abelian, each word map $f: G^{k} \rightarrow G$ is given by a formula of the form $f\left(x_{1}, \ldots, x_{k}\right)=c+\sum_{i=1}^{k} a_{i} x_{i}$, where $c \in G$, $a_{1}, \ldots, a_{k} \in \mathbb{Z}$. If each of the maps $x \mapsto a_{i} x$ has countable range, then so does $f$. If one of them has open range, then (by the Open Mapping Theorem) it is an open map and hence so is $f$.

This characterization was motivated by the following example.

EXAMPLE 3.6. ( $\mathrm{CH})$ Let $n>1$ be an integer and consider the discrete cyclic group $\mathbb{Z}_{n}$. The group $\left(\mathbb{Z}_{n}\right)^{\omega}$ has a dense subgroup which is a Luzin set if and only if $n$ is prime.

Proof. If $n$ is composite with non-trivial factoring $n=a b$, then the map $x \mapsto a x$ maps $\left(\mathbb{Z}_{n}\right)^{\omega}$ into $\{0, a, 2 a, \ldots,(b-1) a\}^{\omega}$. The range is nowhere dense. Furthermore, the fibers of this map are nowhere dense. (The fiber over $a x$ is $\{y: a y=a x\}=\left\{x+b z: z \in\left(\mathbb{Z}_{n}\right)^{\omega}\right\}=x+\{0, b, 2 b, \ldots,(a-1) b\}^{\omega}$.) Now use Corollary 3.5.

If $n$ is prime then the maps $x \mapsto a x$ on $\mathbb{Z}_{n}$ are constant when $a$ is a multiple of $n$, and bijections of $\mathbb{Z}_{n}$ otherwise. The same is thus true for multiplication by $a$ on $\left(\mathbb{Z}_{n}\right)^{\omega}$ and again we can apply Corollary 3.5.

Problem 3.7. Assume the Continuum Hypothesis. Do the following groups have a dense Baire subgroup which has an invariant Borel lifting for its category algebra? (a) $\left(\mathbb{Z}_{4}\right)^{\omega}$, (b) $\left(\mathbb{Z}_{2}\right)^{\omega} \times \mathbb{R}$. 
REMARK 3.8. Analogous examples related to liftings for their measure algebras can be worked out for Haar measures in locally compact Polish groups. We do not elaborate on this here as it is outside the topic of this paper.

\section{Baire separable metric groups}

THEOREM 4.1. It is consistent with ZFC that (1) every second category set in $\mathbb{R}$ has a second category subset of cardinality $\omega_{1}$, and (2) for every Baire separable metric group $(G, d)$ without isolated points, where $d$ is a leftinvariant metric, there are a Borel set $W \subseteq \widetilde{G}$ and a continuous one-to-one map

$$
\varphi:\left(\prod_{i<\omega} m_{i}\right) \times 2^{\omega} \rightarrow \widetilde{G}
$$

where $0<m_{i}<\omega$ for each $i<\omega$ and $(\widetilde{G}, \widetilde{\varrho})$ is given by Theorem 2.6, so that the following properties hold:

(a) $\operatorname{ran} \varphi$ is nowhere dense in $\widetilde{G}$.

(b) $G \cap \operatorname{ran} \varphi$ is a dense Baire subset of $\operatorname{ran} \varphi$.

(c) For any $s \in \prod_{i<\omega} m_{i}$ and $t \in 2^{\omega}$ and any $i<\omega$, there are $\alpha \in G$ and an open neighborhood $S$ of $\varphi(s, t)$ such that $\varphi\left(s, h_{i}(t)\right)=\alpha \varphi(s, t)$ and $S \backslash \operatorname{ran} \varphi$ is partitioned by its intersections with $W$ and $\alpha^{-1} W$.

The proof combines ideas from the proof of Theorem 3.1 and of the result of $[\mathrm{BM}]$ that it is consistent that every second category set of reals is second category in a nowhere dense perfect set. Before proving the theorem, we need some lemmas, principally Lemma 4.5.

Definition 4.2. A sequence

$$
\bar{M}=\left\langle M_{\delta}: \delta \text { is a limit ordinal }\left\langle\omega_{1}\right\rangle\right.
$$

is called an oracle if each $M_{\delta}$ is a countable transitive model of a sufficiently large fragment of ZFC, $\delta \in M_{\delta}, M_{\delta} \models$ " $\delta$ is countable" and for each $A \subseteq \omega_{1}$, $\left\{\delta: A \cap \delta \in M_{\delta}\right\}$ is stationary in $\omega_{1}$.

The meaning of "sufficiently large" depends on the context. In a particular proof, some fragment of ZFC for which models can be produced in ZFC must suffice for all the oracles in the proof. The existence of an oracle is equivalent to $\diamond$ (see [Kun, Theorem II 7.14]), and hence implies CH. We limit the definition of the $\bar{M}$-chain condition to partial orders of cardinality $\omega_{1}$. This covers our present needs. Associated with an oracle $\bar{M}$, there is a filter $\operatorname{Trap}(\bar{M})$ generated by the sets

$$
\left\{\delta<\omega_{1}: \delta \text { is a limit ordinal and } A \cap \delta \in M_{\delta}\right\}, \quad A \subseteq \omega_{1} .
$$

This is a proper normal filter containing all closed unbounded sets. 
Definition 4.3. If $P$ is any partial order, $P^{\prime} \subseteq P$, and $\mathfrak{D}$ is any class of sets, then we write $P^{\prime}<\mathfrak{D} P$ to mean that every predense subset of $P^{\prime}$ which belongs to $\mathfrak{D}$ is predense in $P$.

Definition 4.4. A partial order $P$ satisfies the $\bar{M}$-chain condition, or simply is $\bar{M}$-cc, if there is a one-to-one function $f: P \rightarrow \omega_{1}$ such that

$$
\left\{\delta<\omega_{1}: \delta \text { is a limit ordinal and } f^{-1}(\delta)<_{M_{\delta, f}} P\right\}
$$

belongs to $\operatorname{Trap}(\bar{M})$, where $M_{\delta, f}=\left\{f^{-1}(A): A \subseteq \delta, A \in M_{\delta}\right\}$.

It is not hard to verify that if $P$ is $\bar{M}$-cc, then $P$ is ccc. Also, any oneto-one function $g: P \rightarrow \omega_{1}$ can replace $f$ in the definition.

In what follows, $Q$ denotes the countable forcing notion for adding one Cohen real.

Lemma 4.5 (Main Lemma). Let $\bar{M}=\left\langle M_{\delta}: \delta<\omega_{1}\right\rangle$ be an $\aleph_{1}$-oracle. Let $(G, d)$ be a Baire separable metric group without isolated points, where $d$ is a left-invariant metric. Then there is a forcing notion $P$ which is $\bar{M}-c c$, and $P$-names $\dot{W}$ for a Borel subset of $\widetilde{G}$, and $\dot{m}_{i}, i<\omega$, for non-zero elements of $\omega$, and $\dot{\varphi}$ for a continuous one-to-one map

$$
\dot{\varphi}:\left(\prod_{i<\omega} \dot{m}_{i}\right) \times 2^{\omega} \rightarrow \widetilde{G}
$$

so that for every $\Gamma \subseteq P \times Q$ generic over $V$ and every non-empty open set $B \subseteq \widetilde{G}$,

(a) $\operatorname{ran} \dot{\varphi}[\Gamma]$ is nowhere dense in $\widetilde{G}$,

(b) for any $s \in \prod_{i<\omega} \dot{m}_{i}[\Gamma]$ and $t \in 2^{\omega}$ and any $i<\omega$, there are $\alpha \in G$ and an open neighborhood $S$ of $\dot{\varphi}[\Gamma](s, t)$ such that $\dot{\varphi}[\Gamma]\left(s, h_{i}(t)\right)=$ $\alpha \dot{\varphi}[\Gamma](s, t)$ and $S \backslash \operatorname{ran} \dot{\varphi}[\Gamma]$ is partitioned by its intersections with $\dot{W}[\Gamma]$ and $\alpha^{-1} \dot{W}[\Gamma]$,

and there is no Borel set $X \subseteq \widetilde{G}$ in $V[\Gamma]$ such that

(c) $X \cap \operatorname{ran} \dot{\varphi}[\Gamma]$ is meager relative to $\operatorname{ran} \dot{\varphi}[\Gamma]$,

(d) $B \cap \operatorname{ran} \dot{\varphi}[\Gamma] \neq \emptyset$ and $G \cap B \cap \operatorname{ran} \dot{\varphi}[\Gamma] \subseteq X$.

Note that the non-existence, for each non-empty open $B$, of a Borel set $X$ satisfying (c) and (d) just says that $G \cap \operatorname{ran} \dot{\varphi}[\Gamma]$ is a dense Baire subset of $\operatorname{ran} \dot{\varphi}[\Gamma]$. The given wording is more convenient for our purposes.

Proof of the Main Lemma. Fix a countable dense subgroup $G^{\prime} \subseteq G$ and let $\mathcal{B}$ be the base for $\widetilde{G}$ consisting of $\widetilde{G}$ and all the left translates by elements of $G^{\prime}$ of all the open $\widetilde{\varrho}$-balls of rational radii centered at points of $G^{\prime}$. 
Definition 4.6. Define partial orders $P=P\left(\left\langle a_{\eta}: \eta<\zeta\right\rangle\right)$ where $\zeta \leq \omega_{1}$, $a_{\eta} \in G$ as follows. The conditions in $P$ are the quadruples

$$
\begin{aligned}
p=\left(F_{p},\left\langle\left(U_{p}\right)_{s, t}^{n}: s \in \prod_{i<n} m_{i}^{p}, t\right.\right. & \left.\in 2^{n}, n \leq n_{p}\right\rangle, \\
& \left.\left\langle\alpha_{i}^{p}: i<n_{p}\right\rangle,\left\langle\gamma_{i, j}^{p}: j<m_{i}^{p}, i<n_{p}\right\rangle\right)
\end{aligned}
$$

where:

(1) $e \in F_{p}$ and $F_{p}$ is a finite subset of $G^{\prime} \cup\left\{a_{\eta}: \eta<\zeta\right\}$.

(2) $n_{p}<\omega, 0<m_{i}^{p}<\omega$ for $i<n_{p},\left(U_{p}\right)_{s, t}^{n} \in \mathcal{B}$ for all $s \in \prod_{i<n_{p}} m_{i}^{p}$ and $t \in 2^{n_{p}}, \alpha_{i}^{p}, \gamma_{i, j}^{p} \in G^{\prime}$ and $\gamma_{i, 0}^{p}=e$ for all $j<m_{i}^{p}, i<n_{p}$.

(3) $U_{0,0}^{0}=\widetilde{G}$.

(4) For each $n<n_{p}, s \in \prod_{i<n} m_{i}^{p}, t \in 2^{n}$ and $(k, l) \in m_{n} \times 2$, the following properties hold:

(a) $\left(U_{p}\right)_{s, t}^{n}$ is an open subset of $\widetilde{G}$ and $\operatorname{cl}\left(U_{p}\right)_{s k, t l}^{n+1} \subseteq\left(U_{p}\right)_{s, t}^{n}$, where cl denotes closure in $\widetilde{G}$.

(b) $\left(U_{p}\right)_{s, t}^{n}$ has $\widetilde{\varrho}$-diameter at most $2^{-n}$.

(c) If $\left(k^{\prime}, l^{\prime}\right) \in m_{n} \times 2$ is distinct from $(k, l)$ then $\left(U_{p}\right)_{s k, t l}^{n+1} \cap\left(U_{p}\right)_{s k^{\prime}, t l^{\prime}}^{n+1}$ $=\emptyset$.

(d) $e \in\left(U_{p}\right)_{0,0}^{n}$ and $\left(U_{p}\right)_{s, t}^{n}=\left(\beta_{p}\right)_{s, t}^{n}\left(U_{p}\right)_{0,0}^{n}$, where the subscripts 0 denote the constant zero sequence of length $n$ and

$$
\left(\beta_{p}\right)_{s, t}^{n}=\left(\alpha_{0}^{p}\right)^{t(0)} \gamma_{0, s(0)}^{p} \cdot \ldots \cdot\left(\alpha_{n-1}^{p}\right)^{t(n-1)} \gamma_{n-1, s(n-1)}^{p}
$$

(Take $\left(\beta_{p}\right)_{0,0}^{0}=e$.) The superscripts $t(i)$ indicate exponentiation. All other superscripts are just superscripts.

(5) $F_{p} \subseteq \bigcup\left\{\left(U_{p}\right)_{s, t}^{n_{p}}: s \in \prod_{i<n_{p}} m_{i}^{p}, t \in 2^{n_{p}}\right\}$.

The order on $P$ is: $p \leq q$ if and only if the following conditions are satisfied:

(6) $F_{p} \supseteq F_{q}, n_{p} \geq n_{q}$.

(7) For all $i<n_{q}, m_{i}^{p}=m_{i}^{q}, \alpha_{i}^{p}=\alpha_{i}^{q}$ and, for each $j<m_{i}^{q}, \gamma_{i, j}^{p}=\gamma_{i, j}^{q}$.

(8) For all $n \leq n_{q}, s \in \prod_{i<n} m_{i}^{q}$ and $t \in 2^{n}$, we have $\left(U_{p}\right)_{s, t}^{n}=\left(U_{q}\right)_{s, t}^{n}$.

LEMMA 4.7. For every condition $p$ and every finite set $A \subseteq \bigcup\left\{\left(U_{p}\right)_{s, t}^{n_{p}}\right.$ : $\left.s \in \prod_{i<n_{p}} m_{i}^{p}, t \in 2^{n_{p}}\right\}$, there is a condition $q \leq p$ such that $F_{q}=F_{p}, n_{q}=$ $n_{p}+1, A \subseteq \bigcup\left\{\left(U_{q}\right)_{s, t}^{n_{q}}: s \in \prod_{i<n_{q}} m_{i}^{q}, t \in 2^{n_{q}}\right\}$ and for all $s \in \prod_{i<n_{p}} m_{i}^{p}$, $t \in 2^{n_{p}}$ the set $\bigcup\left\{\left(U_{p}\right)_{s k, t l}^{n_{p}+1}:(k, l) \in m_{n_{p}} \times 2\right\}$ is not dense in $\left(U_{p}\right)_{s, t}^{n_{p}}$.

Proof. Replacing $A$ by $A \cup F_{p}$, we may assume that $F_{p} \subseteq A$. Let $A^{\prime}$ be a finite subset of $\left(U_{p}\right)_{0,0}^{n_{p}}$ containing

$$
\bigcup\left\{\left(\left(\beta_{p}\right)_{s, t}^{n_{p}}\right)^{-1}\left(\left(U_{p}\right)_{s, t}^{n_{p}} \cap A\right): s \in \prod_{i<n_{p}} m_{i}^{p}, t \in 2^{n_{p}}\right\} .
$$


Note that by (1) we have $e \in A$ and hence $e \in A^{\prime}$ (by the first part of (4)(d) and the fact that $\left(\beta_{p}\right)_{0,0}^{n_{p}}=e$ ). Define a condition $q$ by setting $F_{q}=F_{p}$, $n_{q}=n_{p}+1, m_{n_{p}}=\left|A^{\prime}\right|$. Let $q$ agree with $p$ below $n_{p}$ as required by (7), (8). For $x \in A^{\prime}$, choose open sets $S_{x}$ such that $x \in S_{x} \subseteq \operatorname{cl} S_{x} \subseteq\left(U_{p}\right)_{0,0}^{n_{p}}$, the sets $S_{x}, x \in A^{\prime}$, are pairwise disjoint, $\bigcup_{x \in A^{\prime}} S_{x}$ is not dense in $\left(U_{p}\right)_{0,0}^{n_{p}}$, and for all $s \in \prod_{i<n_{p}} m_{i}^{p}$ and $t \in 2^{n_{p}}$, the $\widetilde{\varrho}$-diameter of $\left(\beta_{p}\right)_{s, t}^{n_{p}} S_{x}$ is at most $2^{-n_{q}}$. For all $\alpha \in G$ close enough to $e$, we have $\alpha x \in S_{x}$ for each $x \in A^{\prime}$. Pick one such $\alpha \in G^{\prime} \backslash\{e\}$. For a small enough open neighborhood $W$ of $e$, we derive for each $x \in A^{\prime}$ that $x W$ and $\alpha x W$ are disjoint subsets of $S_{x}$. Choose $B \in \mathcal{B}$ such that $e \in B \subseteq B B \subseteq W$. For each $x \in A^{\prime} \backslash\{e\}$, choose $\gamma_{x} \in G^{\prime}$ close enough to $x$ so that $\gamma_{x}^{-1} x, x^{-1} \gamma_{x} \in B$. We have

$$
x \in \gamma_{x} B \subseteq x B B \subseteq x W .
$$

Let $A^{\prime}=\left\{A^{\prime}(i): i<\left|A^{\prime}\right|\right\}$ with $A^{\prime}(0)=e$. Let $\left(U_{q}\right)_{0,0}^{n_{q}}=B, \alpha_{n_{p}}^{q}=\alpha$, $\gamma_{n_{p}, i}^{q}=\gamma_{A^{\prime}(i)}$ when $0<i<\left|A^{\prime}\right|$ and $\gamma_{n_{p}, 0}^{q}=e$. Define the other values of $\left(U_{q}\right)_{s, t}^{n_{q}}$ by $(4)(\mathrm{d})$. This works.

[We have

$$
\begin{aligned}
\operatorname{cl}\left(U_{q}\right)_{s k, t l}^{n_{p}+1} & =\operatorname{cl}\left(\beta_{q}\right)_{s k, t l}^{n_{p}+1}\left(U_{q}\right)_{0,0}^{n_{p}+1}=\operatorname{cl}\left(\beta_{p}\right)_{s, t}^{n_{p}}\left(\alpha_{n_{p}}^{q}\right)^{l} \gamma_{n_{p}, k}^{q}\left(U_{q}\right)_{0,0}^{n_{p}+1} \\
& =\operatorname{cl}\left(\beta_{p}\right)_{s, t}^{n_{p}} \alpha^{l} \gamma_{n_{p}, k}^{q} B,
\end{aligned}
$$

where $\gamma_{n_{p}, k}^{q}=\gamma_{A^{\prime}(k)}$ if $0<k<\left|A^{\prime}\right|$ and $\gamma_{n_{p}, 0}^{q}=e$. For $x=A^{\prime}(k)$ and $\gamma=\gamma_{n_{p}, k}^{q}$, we have $\gamma B \subseteq x W \subseteq S_{x}$ and $\alpha \gamma B \subseteq \alpha x W \subseteq S_{x}$. From $(*)$ we get

$$
\operatorname{cl}\left(U_{q}\right)_{s k, t l}^{n_{p}+1} \subseteq \operatorname{cl}\left(\beta_{p}\right)_{s, t}^{n_{p}} S_{x}=\left(\beta_{p}\right)_{s, t}^{n_{p}} \operatorname{cl} S_{x} \subseteq\left(\beta_{p}\right)_{s, t}^{n_{p}}\left(U_{p}\right)_{0,0}^{n_{p}}=\left(U_{p}\right)_{s, t}^{n_{p}} .
$$

If $x \in F_{p}$, then $x \in\left(U_{p}\right)_{s, t}^{n_{p}}$ for some $s, t$. Then $y=\left(\left(\beta_{p}\right)_{s, t}^{n_{p}}\right)^{-1} x \in A^{\prime}$. We have $y \in \gamma_{y} B$ and for some $i<\left|A^{\prime}\right|, y=A^{\prime}(i)$. Then $x \in\left(\beta_{p}\right)_{s, t}^{n_{p}} \gamma_{y} B=$ $\left.\left(U_{q}\right)_{s i, t 0}^{n_{p}+1} \cdot\right]$

Let $\dot{K}$ be a $P$-name for the set constructed from the generic filter $\Gamma$ as follows:

$$
\dot{K}=\bigcap_{n<\omega} \bigcup\left\{\left(U_{p}\right)_{s, t}^{n}: p \in \Gamma, n \leq n_{p}\right\} .
$$

(The name really depends on $\bar{a}=\left\langle a_{\eta}: \eta<\zeta\right\rangle$, but omitting explicit mention of $\bar{a}$ should not cause any confusion.)

It will be convenient to have a particularly simple form for the $(P \times Q)$ names $\tau$ for closed sets in $\widetilde{G}$. We will call such a name nice if it is identified with a name for its complement having the form $\bigcup_{B \in \mathcal{B}}\{B\} \times A_{B}$, where the $A_{B}$ 's are countable sets of conditions, $B \subseteq B^{\prime} \Rightarrow A_{B^{\prime}} \subseteq A_{B}$, and given a generic filter $\Gamma, \tau$ names the set whose complement is $\bigcup\left\{B \in \mathcal{B}: \Gamma \cap A_{B}\right.$ $\neq \emptyset$. [For a given name $\tau$, an equivalent nice name can be obtained by 
taking a countable $M \prec H_{\lambda}$ containing $\mathcal{B}$ (for some large enough regular cardinal $\lambda$ ) and setting $A_{B}=M \cap\{x \in P \times Q: x \Vdash B \subseteq \tau\}$.]

Claim 4.8 (Main Claim). Let $P_{\delta}=P\left(\left\langle a_{\eta}: \eta<\delta\right\rangle\right), \delta<\omega_{1}$ be given, as well as a countable $N_{\delta}, P_{\delta} \in N_{\delta}$, a condition $\left(p^{*}, r^{*}\right) \in P_{\delta} \times Q, s \in$ $\prod_{i<n_{p^{*}}} m_{i}^{p^{*}}, t \in 2^{n_{p^{*}}}$ and $P_{\delta} \times Q$-names for closed sets $\tau_{n}$ so that $\Vdash_{P_{\delta} \times Q}$ " $\tau_{n} \cap \dot{K}$ is relatively nowhere dense in $\dot{K}$ ", $n<\omega$. Then we can find $a_{\delta} \in G \cap$ $\left(U_{p^{*}}\right)_{s, t}^{n_{p^{*}}}$ such that, letting $P_{\delta+1}=P\left(\left\langle a_{\eta}: \eta \leq \delta\right\rangle\right)$, the following conditions hold:

(A) Every predense subset of $P_{\delta}$ which belongs to $N_{\delta}$ is a predense subset of $P_{\delta+1}$.

(B) There is a condition $\left(p^{\prime}, r^{\prime}\right) \in P_{\delta+1} \times Q$ extending $\left(p^{*}, r^{*}\right)$ such that $a_{\delta} \in F_{p^{\prime}}$ and for all $n<\omega,\left(p^{\prime}, r^{\prime}\right) \Vdash_{P_{\delta+1} \times Q} a_{\delta} \notin \tau_{n}$.

Proof of the Main Claim. Choose a sufficiently large regular $\lambda$ and choose a countable $N \prec H_{\lambda}$ such that $P_{\delta},\left\langle a_{\eta}: \eta<\delta\right\rangle,\left\langle\tau_{n}: n<\omega\right\rangle, N_{\delta} \in N$. Choose a Cohen real over $N, a_{\delta} \in G \cap\left(U_{p^{*}}\right)_{s, t}^{n_{p^{*}}}$. For the purposes of choosing $a_{\delta}$, think of Cohen forcing as $\mathcal{B}$ ordered by inclusion.

REMARK 4.9. Incompatible elements of $P_{\delta}$ remain incompatible in $P_{\delta+1}$ as incompatibility of two conditions $p, q$ with, say, $n_{p} \leq n_{q}$ can only result from a failure to have $m_{i}^{p}=m_{i}^{q}, \alpha_{i}^{p}=\alpha_{i}^{q}, \gamma_{i, j}^{p}=\gamma_{i, j}^{q},\left(U_{p}\right)_{s, t}^{n}=\left(U_{q}\right)_{s, t}^{n}$ below $n_{p}$ (more precisely for $j<m_{i}, i<n_{p}, s \in \prod_{k<n} m_{k}, t \in 2^{n}, n \leq n_{p}$ ), or a failure of the $\left(U_{q}\right)_{s, t}^{n_{q}}$ to include all points of $F_{p}$.

Proof of condition (A). Let $J \subseteq P_{\delta}$ be predense, $J \in N_{\delta}$. We must show that $J$ is predense in $P_{\delta+1}$. Let $p \in P_{\delta+1}, p \notin P_{\delta}$. By the definition of $P_{\delta+1}$, $F_{p}$ has the form $F \cup\left\{a_{\delta}\right\}$ where $F$ is a finite subset of $\left\{a_{\eta}: \eta<\delta\right\}$. If $p$ is not compatible with any element of $J$, then some $I \in \mathcal{B}$ containing $a_{\delta}$ forces this over $N$. By shrinking $I$, we may take $I \subseteq\left(U_{p^{*}}\right)_{s, t}^{n_{p^{*}}}$. Choose $p_{1} \in P_{\delta}$, a common extension of the condition $\bar{p} \in P_{\delta}$ obtained from $p$ by replacing $F_{p}$ by $F$ and some $p_{2} \in J$ so that for some $s^{\prime} \in \prod_{i<n_{p_{1}}} m_{i}^{p_{1}}, t^{\prime} \in 2^{n_{p_{1}}}$, we have $a_{\delta} \in\left(U_{p_{1}}\right)_{s^{\prime}, t^{\prime}}^{n_{p_{1}}}$. [The set of possible values for $\left(U_{p_{1}}\right)_{s^{\prime}, t^{\prime}}^{n_{p_{1}}}$ covers a dense open subset of $I$ which belongs to $N$, so $a_{\delta}$ belongs to one of them. To see that $I$ is densely covered by these values, fix any $a \in I$ and $\varepsilon>0$. Applying Lemma 4.7 sufficiently many times gives $q \leq \bar{p}$ in $P_{\delta}$ so that some $\left(U_{q}\right)_{s^{\prime \prime}, t^{\prime \prime}}^{n_{q}}$ contains $a$ and has $\widetilde{\varrho}$-diameter less than $\varepsilon$. Then, because $J$ is predense in $P_{\delta}$, we get $p_{1} \in P_{\delta}$, a common extension of $q$ and some $p_{2} \in J$. Then for any $s^{\prime}, t^{\prime}$ for which $\left(U_{p_{1}}\right)_{s^{\prime}, t^{\prime}}^{n_{p_{1}}}$ is contained in $\left(U_{q}\right)_{s^{\prime \prime}, t^{\prime \prime}}^{n_{q}}$, we deduce that the former is within $\varepsilon$ of $a$.] Then $p$ is compatible with $p_{2}$.

Proof of condition (B). Let $p^{\prime}$ be obtained from $p^{*}$ by replacing $F_{p^{*}}$ by $F_{p^{*}} \cup\left\{a_{\delta}\right\}$. Take $r^{\prime}=r^{*}$. If $N\left[a_{\delta}\right]$ believes that these satisfy (B) then they do and we are done. Otherwise, choose $\left(p^{\prime \prime}, r^{\prime \prime}\right)$ in $N\left[a_{\delta}\right]$ extending $\left(p^{\prime}, r^{\prime}\right)$ and 
$i<\omega$ such that

$$
N\left[a_{\delta}\right] \models\left(p^{\prime \prime}, r^{\prime \prime}\right) \Vdash_{P_{\delta+1} \times Q} a_{\delta} \in \tau_{i} .
$$

For some Cohen condition $I \in \mathcal{B}$ containing $a_{\delta}$, we have, letting $x$ be a name for the Cohen real and writing $F_{p^{\prime \prime}}=F^{\prime \prime} \cup\left\{a_{\delta}\right\}$ with $a_{\delta} \notin F^{\prime \prime}$,

$$
N \models I \Vdash " F_{p^{\prime \prime}}=F^{\prime \prime} \cup\{x\} \text { and }\left(p^{\prime \prime}, r^{\prime \prime}\right) \Vdash_{P_{\delta+1} \times Q} x \in \tau_{i} " .
$$

By shrinking $I$, we may assume that it is contained in some $\left(U_{p^{\prime \prime}}\right)_{s^{\prime \prime}, t^{\prime \prime}}^{n_{p^{\prime \prime}} \text {. Let }}$ $\bar{p}^{\prime \prime} \in P_{\delta}$ be obtained from $p^{\prime \prime}$ by replacing $F_{p^{\prime \prime}}$ by $F^{\prime \prime} \cup\{y\}$, where $y$ is any element of $G^{\prime} \cap I$. (Adding $y$ ensures $\bar{p}^{\prime \prime} \Vdash_{P_{\delta}} I \cap \dot{K} \neq \emptyset$.) In $N$, extend $\left(\bar{p}^{\prime \prime}, r^{\prime \prime}\right)$ to $\left(\bar{p}^{\prime \prime \prime}, r^{\prime \prime \prime}\right) \in P_{\delta} \times Q$ which forces (over $\left.P_{\delta} \times Q\right)$ that some $I^{\prime}=\left(U_{\bar{p}^{\prime \prime \prime}}\right)_{s^{\prime \prime \prime}, t^{\prime \prime \prime}}^{n_{\bar{p}^{\prime \prime \prime}}} \subseteq$ $I$ has the property that $I^{\prime} \cap \dot{K} \neq \emptyset$ and $I^{\prime} \cap \dot{K}$ is disjoint from $\tau_{i}$, and choose this extension so that $a_{\delta} \in I^{\prime}$.

[Arranging the last condition is a matter, as in the proof of condition (A), of checking that the set of possible values for $I^{\prime}$, ignoring the requirement $a_{\delta} \in I^{\prime}$, belongs to $N$ and covers a dense subset of $I$. To check the latter, first extend $\bar{p}^{\prime \prime}$ in $N$ to $\widetilde{p} \in P_{\delta}$ so that some $\widetilde{I}=\left(U_{\widetilde{p}}\right)_{\widetilde{s}, \widetilde{t}}^{n_{\tilde{p}}}$ is contained in $I$ and is within a given $\varepsilon$ of a given $a \in I$. Adding an element of $\widetilde{I} \cap G^{\prime}$ to $F_{\widetilde{p}}$ if necessary, we may assume that $\widetilde{p} \Vdash_{P_{\delta}} \widetilde{I} \cap \dot{K} \neq \emptyset$. Next, in $N$, extend $\left(\widetilde{p}, r^{\prime \prime}\right)$ to $\left(\bar{p}^{\prime \prime \prime}, r^{\prime \prime \prime}\right) \in P_{\delta} \times Q$ which decides an $I^{\prime} \in \mathcal{B}$ such that $I^{\prime} \subseteq \widetilde{I}, I^{\prime} \cap \dot{K} \neq \emptyset$ and $I^{\prime} \cap \tau_{i}=\emptyset$. Since $\left(\bar{p}^{\prime \prime \prime}, r^{\prime \prime \prime}\right)$ forces $I^{\prime} \cap \dot{K} \neq \emptyset, I^{\prime}$ meets some $\left(U_{\bar{p}^{\prime \prime \prime}}\right)_{s_{\bar{p}^{\prime \prime \prime}}, t^{\prime \prime \prime}}^{n^{\prime \prime}}$. By adding to $F_{\bar{p}^{\prime \prime \prime}}$ a point $y_{0} \in G^{\prime}$ from the intersection and then extending $\bar{p}^{\prime \prime \prime}$ finitely many times using Lemma 4.7 , we may assume that the $\left(U_{\bar{p}^{\prime \prime \prime}}\right)_{s_{\bar{p}^{\prime \prime \prime}}, t^{\prime \prime \prime}}^{n^{\prime \prime \prime}}$ which contains $y_{0}$ is contained in $I^{\prime}$. Now replace $I^{\prime}$ by $\left.\left(U_{\bar{p}^{\prime \prime \prime}}\right)_{s_{\bar{p}^{\prime \prime \prime}}{ }^{\prime \prime \prime}, t^{\prime \prime \prime}}^{n_{p}}.\right]$

Now let $p^{\prime \prime \prime} \in P_{\delta+1}$ be obtained from $\bar{p}^{\prime \prime \prime}$ by replacing $F_{\bar{p}^{\prime \prime \prime}}$ with $F_{\bar{p}^{\prime \prime \prime}} \cup\left\{a_{\delta}\right\}$. Since $a_{\delta} \in I^{\prime}$,

$$
\left(p^{\prime \prime \prime}, r^{\prime \prime \prime}\right) \Vdash_{P_{\delta+1} \times Q} a_{\delta} \notin \tau_{i} .
$$

More precisely, because

$$
\left(\bar{p}^{\prime \prime \prime}, r^{\prime \prime \prime}\right) \Vdash_{P_{\delta} \times Q} I^{\prime} \cap \tau_{i} \cap \dot{K}=\emptyset
$$

holds in $N\left[a_{\delta}\right]$, it also holds in $V$. (A filter $\Gamma \subseteq P_{\delta} \times Q$ generic over $V$ intersects $N\left[a_{\delta}\right]$ in a filter generic over $N\left[a_{\delta}\right]$, the value $\tau_{i}[\Gamma]$ only depends on this intersection and the statement being forced is absolute.) By an observation of Shelah, it follows that

$$
\left(\bar{p}^{\prime \prime \prime}, r^{\prime \prime \prime}\right) \Vdash_{P_{\delta+1} \times Q} I^{\prime} \cap \tau_{i}=\emptyset .
$$

(See [Bu1993b, Lemma 4.13]. Observe that the proof of (A) showed that $P_{\delta}<_{N} P_{\delta+1}$ and, as noted above, incompatible elements of $P_{\delta}$ remain incompatible in $P_{\delta+1}$.) 
Hence this last statement is true with $p^{\prime \prime \prime}$ in place of $\bar{p}^{\prime \prime \prime}$ as well, and $(*)$ follows. Since $\left(p^{\prime \prime \prime}, r^{\prime \prime \prime}\right)$ extends $\left(p^{\prime \prime}, r^{\prime \prime}\right)$, this contradicts the choice of $\left(p^{\prime \prime}, r^{\prime \prime}\right)$ (using absoluteness again to replace $N\left[a_{\delta}\right]$ by $V$ ). This completes the proof of the Main Claim.

The proof of the Main Lemma is now a mostly routine (for the reader familiar with oracle-cc forcing) bookkeeping argument using the Main Claim. There are a few points where some care is needed, so we give the argument. The middle part of the argument closely follows [Bu1993b, pp. 144-145].

Suppose $\delta \leq \omega_{1}$ and $\tau$ is a nice $P \times Q$-name for a closed set in $\widetilde{G}$. If $\tau=\bigcup_{B \in \mathcal{B}}\{B\} \times A_{B}$ is such that the $A_{B}$ 's are contained in $P_{\delta} \times Q$, then $\tau$ is also a $P_{\delta} \times Q$-name for a closed set.

FACT 4.10. If $\Vdash_{P \times Q}$ " $\tau$ is a nowhere dense subset of $\dot{K}$ ", then also $\Vdash_{P_{\delta} \times Q}$ " $\tau$ is a nowhere dense subset of $\dot{K}$ ".

(As pointed out after the definition of $\dot{K}$, this notation denotes two different names in the two statements above.)

Claim 4.11. If $y \in B$ and $u \Vdash_{P_{\delta} \times Q} B \subseteq \tau^{\mathrm{c}}$ then $u$ is compatible with some $v \in A_{B^{\prime}}$ for some $B^{\prime}$ so that $y \in B^{\prime} \subseteq B$.

[Let $\Gamma$ be a generic filter containing $u$. Then $y \in B \subseteq \tau[\Gamma]^{\mathrm{c}}=\bigcup\left\{B^{\prime}\right.$ : $\left.B^{\prime} \in \mathcal{B}, \Gamma \cap A_{B^{\prime}} \neq \emptyset\right\}$. Hence, there is $v \in \Gamma \cap A_{B^{\prime}}$ for some $B^{\prime}$ such that $y \in B^{\prime}$. Choose $B^{\prime \prime} \in \mathcal{B}$ so that $y \in B^{\prime \prime} \subseteq B \cap B^{\prime}$. By assumption, $A_{B^{\prime}} \subseteq A_{B^{\prime \prime}}$ so that $v \in A_{B^{\prime \prime}}$. Also, $y \in B^{\prime \prime} \subseteq B$.]

To prove Fact 4.10 , suppose it fails. Then, for some condition $(p, r) \in$ $P_{\delta} \times Q$ and some $B \in \mathcal{B}$,

$$
(p, r) \Vdash_{P_{\delta} \times Q} \emptyset \neq B \cap \dot{K} \subseteq \tau .
$$

Then some extension $\left(p^{\prime}, r^{\prime}\right) \in P \times Q$ decides $B_{0} \in \mathcal{B}, B_{0} \subseteq B$ meeting $\dot{K}$ but disjoint from $\tau$. Note that $B_{0}$ must meet some $\left(U_{p^{\prime}}\right)_{s, t}^{n_{p^{\prime}}}$. We may assume that there is a point $y \in G^{\prime} \cap B_{0} \cap\left(U_{p^{\prime}}\right)_{s, t}^{n_{p^{\prime}}}$ in $F_{p^{\prime}}$ (by adding one if necessary). By Claim 4.11, $\left(p^{\prime}, r^{\prime}\right)$ is compatible with some $\left(p^{\prime \prime}, r^{\prime \prime}\right) \in A_{B_{1}}$ for some $B_{1}$ such that $y \in B_{1} \subseteq B_{0}$. Then $(p, r)$ modified by adding $y$ to $F_{p}$ is also compatible with $\left(p^{\prime \prime}, r^{\prime \prime}\right)$. As observed earlier, this modification of $(p, r)$ and $\left(p^{\prime \prime}, r^{\prime \prime}\right)$ must have a common extension $(\bar{p}, \bar{r})$ in $P_{\delta} \times Q$. Since $y \in F_{\bar{p}}$, we have $(\bar{p}, \bar{r}) \Vdash_{P_{\delta} \times Q} B_{1} \cap \dot{K} \neq \emptyset$. However, $(\bar{p}, \bar{r}) \leq\left(p^{\prime \prime}, r^{\prime \prime}\right) \in A_{B_{1}}$, so $(\bar{p}, \bar{r}) \Vdash_{P_{\delta} \times Q} B_{1} \cap \tau=\emptyset$, contradicting $(* *)$ and the facts that $B_{1} \subseteq B$ and $(\bar{p}, \bar{r}) \leq(p, r)$. This proves Fact 4.10 .

Fix a bijection $g: \omega \rightarrow \mathcal{B}$. Let $\left\langle\left(p_{\delta}, s_{\delta}, t_{\delta}, r_{\delta}, \bar{\sigma}_{\delta}\right): \delta<\omega_{1}\right\rangle$ list all 5 -tuples $(p, s, t, r, \bar{\sigma})$ where $p<\omega_{1}, s \in \omega^{<\omega}, t \in 2^{<\omega}, r \in Q, \bar{\sigma}=\left\langle\sigma_{i}: i<\omega\right\rangle$, $\sigma_{i}=\bigcup_{n}\{n\} \times A_{n}\left(\sigma_{i}\right)$, and each $A_{n}\left(\sigma_{i}\right)$ is a countable subset of $\omega_{1} \times Q$. We can arrange that for each $\delta, p_{\delta}<\delta$ and, for all $n, i, A_{n}\left(\left(\sigma_{\delta}\right)_{i}\right) \subseteq \delta \times Q$. We also want that each 5 -tuple $(p, s, t, r, \bar{\sigma})$ is listed sufficiently often so that 
$\left\{\delta<\omega_{1}:(p, s, t, r, \bar{\sigma})=\left(p_{\delta}, s_{\delta}, t_{\delta}, r_{\delta}, \bar{\sigma}_{\delta}\right)\right\}$ meets every member of $\operatorname{Trap}(\bar{M})$. [Apply [Kun, Theorem II.6.11] to the dual ideal of $\operatorname{Trap}(\bar{M})$.] For $\delta<\omega_{1}$, inductively define models $N_{\delta},\left\langle\bar{a}_{\eta}: \eta<\delta\right\rangle$, and a 1-1 function $h_{\delta}: P_{\delta} \rightarrow \omega_{1}$ whose range is an initial segment of $\omega_{1}$ which includes $\delta$. As in the statement of the Main Claim we write $P_{\delta}=P\left(\left\langle\bar{a}_{\eta}: \eta<\delta\right\rangle\right)$. We want $\forall \delta^{\prime}<\delta, h_{\delta^{\prime}} \subseteq h_{\delta}$, and at limits, $h_{\delta}=\bigcup_{\delta^{\prime}<\delta} h_{\delta^{\prime}}$ and $P_{\delta}=\bigcup_{\delta^{\prime}<\delta} P_{\delta^{\prime}}$. At successor stages use the Main Claim to get $P_{\delta+1}$, with:

(i) $p^{*}=h_{\delta}^{-1}\left(p_{\delta}\right)$.

(ii) If $s_{\delta} \in \prod_{i<n_{p^{*}}} m_{i}^{p^{*}}$ and $t_{\delta} \in 2^{n_{p^{*}}}$, then $(s, t)=\left(s_{\delta}, t_{\delta}\right)$. Otherwise $(s, t)=(0,0)$ where the 0 -sequences have length $n_{p^{*}}$.

(iii) $r^{*}=r_{\delta}$.

(iv) $\tau_{i}=\bigcup_{B}\{B\} \times\left\{\left(h_{\delta}^{-1}(\alpha), q\right):\left(g^{-1}(B),(\alpha, q)\right) \in\left(\sigma_{\delta}\right)_{i}, \alpha<\omega_{1}\right\}$, $i<\omega$.

(It does not matter here whether $\tau_{i}$ is a nice name in the sense defined earlier. It simply names the set whose complement is defined from $\Gamma$ by $\bigcup\left\{B \in \mathcal{B}: \exists u \in \Gamma,(B, u) \in \tau_{i}\right\}$.)

(v) $N_{\delta}=$ a countable transitive model of a large fragment of ZFC such that $N_{\delta} \supseteq \bigcup_{\delta^{\prime}<\delta} N_{\delta^{\prime}} \cup M_{\delta, h_{\delta}} \cup\{\bar{\tau}\} \cup\left\{P_{\delta}\right\}$.

Let $P=\bigcup_{\delta<\omega_{1}} P_{\delta}, h=\bigcup_{\delta<\omega_{1}} h_{\delta}$. Define $\dot{K}$ for $P$ as above. We must first show that $P$ is $\bar{M}$-cc. By induction on $\alpha>\delta$ we easily deduce from (A) of the Main Claim that

$$
P_{\delta}<M_{\delta, h} P_{\alpha}
$$

for every $\alpha<\omega_{1}$, and hence $P_{\delta}<_{M_{\delta, h}} P$. For a closed unbounded set of $\delta$, we will have $P_{\delta}=h^{-1}(\delta)$. Thus $h$ witnesses that $P$ is $\bar{M}$-cc.

Now, let $\Gamma \subseteq P \times Q$ be generic over $V$. Let $m_{i}, \alpha_{i}, \gamma_{i, j}\left(j<m_{i}, i<\omega\right)$, $U_{s, t}^{n}\left(n<\omega, s \in \prod_{i<n} m_{i}, t \in 2^{n}\right)$ denote, respectively, $m_{i}^{p}, \alpha_{i}^{p}, \gamma_{i, j}^{p},\left(U_{p}\right)_{s, t}^{n}$ for any $p \in \Gamma$ for which $i<n \leq n_{p}$. Lemma 4.7 ensures that there are arbitrarily large values of $n_{p}$ for $p \in \Gamma$. Define $\varphi$ : $\left(\prod_{i<\omega} m_{i}\right) \times 2^{\omega} \rightarrow \widetilde{G}$ by $\{\varphi(s, t)\}=\bigcap_{n<\omega} U_{s\lceil n, t \uparrow n}^{n}$. By Definition 4.6(4)(a,b,c), $\varphi$ is a well-defined one-to-one continuous map.

Part (a) of the Main Lemma follows from (4)(b) and a simple genericity argument using Lemma 4.7. (Cf. the reason that $C$ is nowhere dense in $G$ in the proof of Theorem 3.1.) Part (b) follows by an argument similar to the end of the proof of Theorem 3.1. For $x \in G \backslash K$, there is a largest $n=n(x)<\omega$ such that $x \in U_{s, t}^{n}$ for some (unique) $s=s(x) \in \prod_{i<n} m_{i}, t=t(x) \in 2^{n}$. Define

$$
W=\left\{x \in G \backslash K: t_{0}+\cdots+t_{n-1} \text { is odd }\right\} .
$$

Then $W$ is the union of countably many sets of the form $U_{s, t}^{n} \backslash \bigcup\left\{U_{s k, t i}^{n+1}\right.$ : $\left.k<m_{n}, i<2\right\}$ and hence is Borel. If $i<n<\omega$ and $t \in 2^{\omega}$ satisfies $t(i)=0$, 
then by $(4)(\mathrm{d})$ we have, for each $s \in \prod_{i<\omega} m_{i}$,

$$
U_{s\left\lceil n, h_{i}(t)\lceil n\right.}^{n}=\beta_{s \uparrow i, t \uparrow i}^{i} \alpha_{i}\left(\beta_{s \uparrow i, t \uparrow i}^{i}\right)^{-1} U_{s\lceil n, t \uparrow n}^{n} .
$$

Similarly, if $t(i)=1$, then

$$
U_{s\left\lceil n, h_{i}(t) \uparrow n\right.}^{n}=\beta_{s\lceil i, t \uparrow i}^{i} \alpha_{i}^{-1}\left(\beta_{s \uparrow i, t \uparrow i}^{i}\right)^{-1} U_{s\lceil n, t \uparrow n}^{n} .
$$

Claim 4.12. For any $s \in \prod_{i<\omega} m_{i}, t \in 2^{\omega}$ and any $i<\omega$, there is an open neighborhood $S$ of $\varphi(s, t)$ and there is an $\alpha \in G$ such that $\varphi\left(s, h_{i}(t)\right)=$ $\alpha \varphi(s, t)$. Furthermore, $S \backslash K$ is partitioned by its intersections with $W$ and $\alpha^{-1} W$.

Proof. We can take for $S$ any $U_{s\lceil m, t \uparrow m}^{m}$ with $i<m$. Intersecting over $n$ in the two displayed equations above shows that $\varphi\left(s, h_{i}(t)\right)=\alpha \varphi(s, t)$ where $\alpha=\beta_{s \uparrow i, t \uparrow i}^{i} \alpha_{i}^{ \pm 1}\left(\beta_{s \uparrow i, t \uparrow i}^{i}\right)^{-1}$. If $x \in S \backslash K=U_{s \uparrow m, t \uparrow m}^{m} \backslash K$, then from the definition of $\alpha$ it follows that precisely one of $x, \alpha x$ belongs to $W$. This gives the second statement of the claim.

Suppose there were a generic $\Gamma \subseteq P \times Q, B \in \mathcal{B}$ and a Borel set $X \in V[\Gamma]$ which give a counterexample to the Main Lemma. Then there is a sequence of nice $P \times Q$-names $\left\langle\tau_{i}: i<\omega\right\rangle$ for closed sets whose traces on $\dot{K}$ are relatively nowhere dense such that $X \cap \dot{K} \subseteq \bigcup_{i<\omega} \tau_{i}[\Gamma]$, and there is a condition $\left(p^{*}, r^{*}\right) \in P \times Q$ which forces $(\mathrm{d})$ of the Main Lemma with $\bigcup_{i<\omega} \tau_{i}$ in place of $X$, i.e., $B \cap \dot{K} \neq \emptyset$ and $G \cap B \cap \dot{K} \subseteq \bigcup_{i<\omega} \tau_{i}$. The first statement implies that $B$ meets at least one $\left(U_{p^{*}}\right)_{s, t}^{n_{p^{*}}}$. By adding a point of $G^{\prime}$ from such an intersection to $F_{p^{*}}$ and extending $p^{*}$ finitely many times using Lemma 4.7, we can assume that $\left(U_{p^{*}}\right)_{s, t}^{n_{p^{*}}} \subseteq B$. Then $B$ can be replaced by $\left(U_{p^{*}}\right)_{s, t}^{n_{p^{*}}}$.

The set of all $\delta<\omega_{1}$ which satisfy any one of the following conditions belongs to $\operatorname{Trap}(\bar{M})$ :

(i) $\left(p^{*}, r^{*}\right) \in P_{\delta} \times Q$.

(ii) $\tau_{i}=\bigcup_{B \in \mathcal{B}}\{B\} \times A_{B}\left(\tau_{i}\right)$ is a $P_{\delta} \times Q$-name, $i<\omega$.

(iii) $h^{-1}(\delta)=P_{\delta}$.

(iv) $\sigma_{i}=\bigcup_{n}\{n\} \times\left\{(h(p), q):(p, q) \in A_{g(n)}\left(\tau_{i}\right)\right\} \in M_{\delta}, i<\omega$.

(v) For all $\delta^{\prime} \geq \delta$ and $i<\omega, \Vdash_{P_{\delta^{\prime}} \times Q}$ " $\tau_{i}$ is a closed set which has a relatively nowhere dense trace on $\dot{K}$ ".

[As explained above, for $(\mathrm{v})$ all that is required is to choose $\delta$ large enough so that each $\tau_{i}$ is a $P_{\delta} \times Q$-name.]

Choose a $\delta$ satisfying all these properties and for which $\left(p_{\delta}, s_{\delta}, t_{\delta}, r_{\delta}, \bar{\sigma}_{\delta}\right)$ $=\left(p^{*}, s, t, r^{*}, \bar{\sigma}\right)$. Then $\bar{\tau}_{\delta}$ is a sequence of $P_{\delta} \times Q$-names for closed sets which have a relatively nowhere dense trace on $\dot{K}$. So by (B) of the Main Claim, there is a condition $\left(p^{\prime}, r^{\prime}\right) \leq\left(p^{*}, r^{*}\right)$ with the property given there. This will clearly contradict our choice of $\left(p^{*}, r^{*}\right)$ if we can show that the subscript $P_{\delta+1} \times Q$ in (B) can be replaced with $P \times Q$. Fix $i<\omega$. By induction 
on $\alpha>\delta$ we have $P_{\delta+1}<_{N_{\delta+1}} P_{\alpha}$ and hence $P_{\delta+1}<_{N_{\delta+1}} P$. As already observed, elements of $P_{\delta+1}$ which are incompatible remain incompatible in $P$. From Shelah's observation mentioned above [Bu1993b, Lemma 4.13], we have $\left(p^{\prime}, r^{\prime}\right) \Vdash_{P \times Q} a_{\delta} \notin \tau_{i}$, which is the desired contradiction. This proves the Main Lemma.

Before turning to the proof of Theorem 4.1, we recall the basic properties of oracle-cc forcing. See [Sh1998, Chapter IV] for the details. A version of this material is also explained in [Bu1993b, Sections 4-6].

Proposition 4.13. The $\bar{M}$-cc has the following properties:

(1) If $\alpha<\omega_{2}$ is a limit ordinal, $\left\langle\left\langle P_{\beta}\right\rangle_{\beta \leq \alpha},\left\langle\dot{Q}_{\beta}\right\rangle_{\beta<\alpha}\right\rangle$ is a finite-support $\alpha$-stage iteration of partial orders, and for each $\beta<\alpha, P_{\beta}$ is $\bar{M}$-cc, then $P_{\alpha}$ is $\bar{M}-c c$.

(2) If $P$ is $\bar{M}$-cc, then there is a $P$-name $\bar{M}^{*}$ for an oracle such that for each $P$-name $\dot{Q}$ for a partial order, if $\Vdash_{P}$ " $\dot{Q}$ is $\bar{M}^{*}$-cc" then $P * \dot{Q}$ is $\bar{M}-c c$.

(3) If $\bar{M}_{\alpha}, \alpha<\omega_{1}$, are oracles, then there is an oracle $\bar{M}$ such that for any partial order $P$, if $P$ is $\bar{M}$-cc, then $P$ is $\bar{M}_{\alpha}-c c$ for all $\alpha<\omega_{1}$.

Lemma 4.14. Assume $\diamond$. Let $A$ be a non-meager subset of $\mathbb{R}$. Then there is an oracle $\bar{M}=\left\langle M_{\delta}: \delta<\omega_{1}\right\rangle$ such that if $P$ is any $\bar{M}$-cc partial order, then $\Vdash_{P}$ " $A$ is non-meager".

Proof. This is [Sh1998, Example IV.2.2].

We now prove Theorem 4.1. Because of part (1) of its statement, it suffices to establish part (2) for groups of cardinality $\omega_{1}$. [If $(G, d)$ is arbitrary, then the fact that $\widetilde{G}$ has a dense $G_{\delta}$ subset homeomorphic to $\omega^{\omega}$ ensures that $G$ has a dense subgroup $G_{0}$ of size $\omega_{1}$ which is Baire. (Take a second category set of size $\omega_{1}$ in each non-empty member of a countable base for $G$ and let $G_{0}$ be the subgroup generated by their union.) Then any $V, \varphi$ which work for $\left(G_{0}, d \uparrow\left(G_{0} \times G_{0}\right)\right)$ will continue to work for $(G, d)$ since $\widetilde{G}=\widetilde{G}_{0}$ and (b), (c) are preserved by enlarging $G$ inside $\widetilde{G}$.]

Start with a ground model of $V=L$. Fix a diamond sequence

$$
\left\langle\left(f_{\alpha}, g_{\alpha}, h_{\alpha}\right): \alpha<\omega_{2}, \operatorname{cof}(\alpha)=\omega_{1}\right\rangle
$$

for trapping triples $(f, g, h)$ consisting of:

(1) A function $f: \omega_{2} \rightarrow\left(\left[\omega_{2}\right]^{\leq \omega}\right)^{\omega}$. The idea of $f$ is that, with $\omega_{2}$ identified with the ccc partial order we are about to build, $\left[\omega_{2}\right] \leq \omega$ contains the maximal antichains. Thus, $\left(\left[\omega_{2}\right]^{\leq \omega}\right)^{\omega}$ contains a name for each real number (construed as a subset of $\omega$ ). Then for any nonmeager set $X$ in the extension, we can find a ground model function $f: \omega_{2} \rightarrow\left(\left[\omega_{2}\right]^{\leq \omega}\right)^{\omega}$ enumerating the names of the elements of $X$. 
(2) Functions $g: \omega_{1} \times \omega_{1} \rightarrow\left(\left[\omega_{2}\right]^{\leq \omega}\right)^{\omega \times \omega}$ and $h: \omega_{1} \times \omega_{1} \rightarrow\left(\left[\omega_{2}\right]^{\leq \omega}\right)^{\omega}$ intended to represent a pair $(G, d)$ where $G$ is a group of cardinality $\omega_{1}$ whose underlying set we take to be $\omega_{1}$ itself and $d$ is a left-invariant metric for $G$. More specifically, $h(\alpha, \beta)$ is intended to represent (the name of ) the value $d(\alpha, \beta)$ and $g(\alpha, \beta)$ is intended to represent the composition of $\alpha$ and $\beta$ under the group operation. (If the composition of $\alpha$ and $\beta$ is $\gamma$, then $g(\alpha, \beta)$ can be thought of as a real which is a subset of $\omega \times \omega$ and which is a well-ordering of $\omega$ with order-type $\gamma$.)

So for each $\alpha<\omega_{2}$ of cofinality $\omega_{1}, f_{\alpha}: \alpha \rightarrow([\alpha] \leq \omega) \omega, g_{\alpha}: \omega_{1} \times \omega_{1} \rightarrow$ $\left([\alpha]^{\leq \omega}\right)^{\omega \times \omega}$ and $h_{\alpha}: \omega_{1} \times \omega_{1} \rightarrow\left([\alpha]^{\leq \omega}\right)^{\omega}$. Also, for each $(f, g, h)$ as in (1)(2), $\left\{\alpha<\omega_{2}: \operatorname{cof}(\alpha)=\omega_{1}, f\left\lceil\alpha=f_{\alpha}, g=g_{\alpha}\right.\right.$ and $\left.h=h_{\alpha}\right\}$ is stationary in $\omega_{2}$.

We will inductively define an $\omega_{2}$-stage finite support iteration

$$
\left\langle\left\langle P_{\alpha}\right\rangle_{\alpha \leq \omega_{2}},\left\langle\dot{Q}_{\alpha}\right\rangle_{\alpha<\omega_{1}}\right\rangle
$$

as well as $P_{\alpha}$-names $\bar{M}_{\alpha}$ for oracles and one-to-one functions $F_{\alpha}: P_{\alpha} \rightarrow \omega_{2}$ for $\alpha<\omega_{2}$ such that the range of each $F_{\alpha}$ is an initial segment of $\omega_{2}$ which includes $\alpha$, and for $\beta<\alpha<\omega_{2}$, we have $F_{\beta} \subseteq F_{\alpha}$. (At each stage, $F_{\alpha}$ is any function satisfying these conditions.)

For $\alpha<\omega_{2}$, we will let $\dot{X}_{\alpha}$ denote the $P_{\alpha}$-name for the set of real numbers whose elements have the names

$$
\bigcup_{n<\omega}\{n\} \times F_{\alpha}^{-1}\left(f_{\alpha}(\xi)(n)\right), \quad \xi<\alpha .
$$

Similarly, we will let $\dot{G}_{\alpha}$ and $\dot{d}_{\alpha}$ denote the $\omega_{1} \times \omega_{1}$-sequences of $P_{\alpha}$-names for real numbers

$$
\left\langle\bigcup_{(m, n) \in \omega \times \omega}\{(m, n)\} \times F_{\alpha}^{-1}\left(g_{\alpha}\left(\xi_{1}, \xi_{2}\right)(m, n)\right): \xi_{1}, \xi_{2}<\omega_{1}\right\rangle
$$

and

$$
\left\langle\bigcup_{n<\omega}\{n\} \times F_{\alpha}^{-1}\left(h_{\alpha}\left(\xi_{1}, \xi_{2}\right)(n)\right): \xi_{1}, \xi_{2}<\omega_{1}\right\rangle
$$

respectively. At stage $\alpha<\omega_{2}$ of the construction, if $\operatorname{cof}(\alpha)=\omega_{1}$ and if

$$
\Vdash_{P_{\alpha}} \text { “唋 is not meager", }
$$

then we use Lemma 4.14 to get a $P_{\alpha}$-name $\bar{M}_{\alpha}^{\prime}$ for an oracle so that if $P$ is any forcing notion which is $\bar{M}_{\alpha}^{\prime}$-cc, then $X_{\alpha}$ remains non-meager after forcing with $P$.

If $\operatorname{cof}(\alpha)=\omega_{1}$ and if

$\Vdash_{P_{\alpha}} "\left(\dot{G}_{\alpha}, \dot{d}_{\alpha}\right)$ is a Baire separable metric group with left-invariant metric $\dot{d}_{\alpha} "$, 
then use Lemma 4.5 to get a $P_{\alpha}$-name $\dot{Q}_{\alpha}$ for a partial order which is $\bar{M}_{\alpha}$-cc and forces a continuous function $\varphi$ and a Borel set $V$ as described in the statement of the lemma. Then use Lemma 4.14 and Proposition 4.13(3) to get a $P_{\alpha+1}$-name $\bar{M}_{\alpha+1}^{\prime}$ for an oracle such that if $P$ is any forcing notion which is $\bar{M}_{\alpha+1}^{\prime}$-cc, then $G_{\alpha} \cap \operatorname{ran} \varphi$ remains a dense Baire subset of $\operatorname{ran} \varphi$ after forcing with $P$.

Otherwise, let $\bar{M}_{\alpha}^{\prime}$ be any $P_{\alpha}$-name for an oracle.

For $\beta<\alpha$, let $P_{\beta \alpha}$ be the usual $P_{\beta}$-name for a partial order such that $P_{\alpha}$ is isomorphic to a dense subset of $P_{\beta} * P_{\beta \alpha}$ (see [Ba]). Let $\bar{M}_{\beta \alpha}$ be a $P_{\alpha}$-name for an oracle such that

$$
\begin{aligned}
& \text { if } \Vdash_{P_{\beta}} \text { " } P_{\beta, \alpha} \text { is } \bar{M}_{\beta} \text {-cc and } \Vdash_{P_{\beta, \alpha}} \text { " } \dot{Q}_{\alpha} \text { is } \bar{M}_{\beta \alpha} \text {-cc" ", } \\
& \text { then } \Vdash_{P_{\beta}} \text { " } P_{\beta, \alpha+1}=P_{\beta, \alpha} * \dot{Q}_{\alpha} \text { is } \bar{M}_{\beta} \text {-cc". }
\end{aligned}
$$

(There is such an $\bar{M}_{\beta \alpha}$ by Proposition $4.13(2)$. In (1), $\bar{M}_{\beta \alpha}$ is actually a $P_{\beta^{-}}$ name for a $P_{\beta, \alpha}$-name for an oracle. We denote the corresponding $P_{\alpha}$-name also by $\bar{M}_{\beta \alpha}$.)

Let $\bar{M}_{\alpha}$ be a $P_{\alpha}$-name for an oracle such that

$$
\Vdash_{P_{\alpha}} \text { "If } \dot{Q}_{\alpha} \text { is } \bar{M}_{\alpha} \text {-cc, then } \dot{Q}_{\alpha} \text { is } \bar{M}_{\alpha}^{\prime} \text {-cc and } \bar{M}_{\beta \alpha^{-c c}} \text { for all } \beta<\alpha \text { ". }
$$

(Use Proposition 4.13(3).)

In all other cases, take $\dot{Q}_{\alpha}$ to name the partial order $Q$ for adding one Cohen real. We thus have

$$
\Vdash_{P_{\alpha}} \text { " } \dot{Q}_{\alpha} \text { is } \bar{M}_{\alpha} \text {-cc". }
$$

Now suppose that for some $P_{\omega_{2}}$-name $\dot{X}$ we have

$$
\Vdash_{P_{\omega_{2}}} \text { " } \dot{X} \text { is not meager". }
$$

(Every non-meager set in any extension has a name forced by the weakest condition to be non-meager since there always is a non-meager set.) Fix a name $\dot{f}$ such that

$$
\Vdash_{P_{\omega_{2}}} \text { " } \dot{f}: \omega_{2} \rightarrow \dot{X} \text { is onto". }
$$

Then define $f: \omega_{2} \rightarrow\left(\left[\omega_{2}\right]^{\leq \omega}\right)^{\omega}$ so that if

$$
\tau_{\xi}=\bigcup_{n<\omega}\{n\} \times F^{-1}(f(\xi)(n)), \quad \xi<\omega_{2},
$$

then, for each $\xi<\omega_{2}$,

$$
\Vdash_{P_{\omega_{2}}} \dot{f}(\xi)=\tau_{\xi} .
$$

There is a closed unbounded set $C \subseteq \omega_{2}$ such that for each $\alpha \in C$ of cofinality $\omega_{1}$ we have:

(i) $f\left\lceil\alpha: \alpha \rightarrow\left([\alpha]^{\leq \omega}\right)^{\omega}\right.$.

(ii) $\forall \xi<\alpha, \tau_{\xi}$ is a $P_{\alpha}$-name.

(iii) $\vdash_{P_{\alpha}}$ " $\left\{\tau_{\xi}: \xi<\alpha\right\}$ is not meager". 
(For (iii), note that when $\alpha$ has cofinality $\omega_{1}$, each $P_{\alpha}$-name for a meager set is a $P_{\beta}$-name for some $\beta<\alpha$. Thus, if $M$ is an elementary submodel of $H_{\theta}$ for a suitably large $\theta$ such that $|M|=\omega_{1}, M^{\omega} \subseteq M,\left\langle\tau_{\xi}: \xi<\omega_{2}\right\rangle \in M$ and $\alpha=M \cap \omega_{2} \in \omega_{2}$ has cofinality $\omega_{1}$, then for each (nice) $P_{\alpha}$-name $\sigma$ for a meager Borel set, we have $\sigma \in M$ and hence $M$ knows about a maximal antichain of conditions each deciding a $\xi$ for which $\tau_{\xi}$ is forced not to be in $\sigma$. The antichain is countable and hence contained in $M$. For each condition in the antichain, the least $\xi$ which it decides is in $M$ and hence below $\alpha$. Hence $\Vdash_{P_{\alpha}}$ " $\left\{\tau_{\xi}: \xi<\alpha\right\}$ is not contained in $\sigma$ ".)

Choose such an $\alpha$ of cofinality $\omega_{1}$ for which $f\left\lceil\alpha=f_{\alpha}\right.$. By (i) and (ii), the definition of $\tau_{\xi}$ would not change if we used $f_{\alpha}$ instead of $f$ and $F_{\alpha}$ instead of $F$. Then from the definition of $\dot{X}_{\alpha}$ we get

$$
\Vdash_{P_{\alpha}} \dot{X}_{\alpha}=\left\{\tau_{\xi}: \xi<\alpha\right\} .
$$

So at stage $\alpha$ we chose a $P_{\alpha}$-name $\bar{M}_{\alpha}$ and we arrange that

$$
\Vdash_{P_{\alpha}} \text { " } P_{\alpha, \gamma} \text { is } \bar{M}_{\alpha} \text {-cc". }
$$

[This follows easily by induction on $\gamma \geq \alpha$ and Proposition 4.13(1,2). (Recall that $P_{\alpha, \gamma}$ can be viewed in the canonical way as an iteration: see [Ba].) At limits $\gamma$ use Proposition 4.13(1). At stages $\gamma+1$, use (3) to get $\vdash_{P_{\gamma}}$ " $\dot{Q}_{\gamma}$ is $\bar{M}_{\gamma}$-cc" and then use (2) and (1) with $(\beta, \alpha)$ replaced by $(\alpha, \gamma)$.]

Hence, by the choice of $\bar{M}_{\alpha}$,

$$
\Vdash_{P_{\alpha}} \Vdash_{P_{\alpha, \gamma}} \text { " } \dot{X}_{\alpha} \text { is not meager", }
$$

from which it follows that

$$
\Vdash_{P_{\alpha}} \Vdash_{P_{\alpha, \omega_{2}}} \text { " } \dot{X}_{\alpha} \text { is not meager" }
$$

since if this failed then we would have

$$
p \Vdash_{P_{\alpha}} q \Vdash_{P_{\alpha, \omega_{2}}} \dot{X}_{\alpha} \subseteq \dot{B}
$$

for some conditions $p \in P_{\alpha}, q \in P_{\alpha, \omega_{2}}$ and some name $\dot{B}$ for a meager Borel set. But then for some $\gamma$, we have $\alpha<\gamma<\omega_{2}, q \in P_{\alpha, \gamma}$ and $\dot{B}$ is a $P_{\gamma}$-name, and this contradicts (4).

By what we have established, there are guaranteed to be sets of cardinality $\omega_{1}$ which are not meager in any extension by $P_{\omega_{2}}$. Hence there are guaranteed to be Baire separable metric groups of cardinality $\omega_{1}$ (for example, subgroups of $\mathbb{R}$ ). Suppose that for some $P_{\omega_{2}}$-names $\dot{G}$ and $\dot{d}$ and a nice $P_{\omega_{2}}$-name $\dot{f}$ for a subset of $\omega \times \omega_{1}$, we have

$\Vdash_{P_{\omega_{2}}}$ " $(\dot{G}, \dot{d})$ is a Baire separable metric group with underlying set $\omega_{1}$ (where $\dot{G}$ names the operation) and $\dot{f}: \omega \rightarrow \omega_{1}$ has dense range in $(\dot{G}, \dot{d})$ ". 
(By what we just said, every Baire separable metric group with underlying set $\omega_{1}$ has a name such that the weakest condition forces the desired properties.)

Define $g: \omega_{1} \times \omega_{1} \rightarrow\left(\left[\omega_{2}\right]^{\leq \omega}\right)^{\omega \times \omega}$ and $h: \omega_{1} \times \omega_{1} \rightarrow\left(\left[\omega_{2}\right]^{\leq \omega}\right)^{\omega}$ so that if

$$
\begin{aligned}
& \underline{\dot{G}}\left(\xi_{1}, \xi_{2}\right)=\bigcup_{(m, n) \in \omega \times \omega}\{(m, n)\} \times F^{-1}\left(g\left(\xi_{1}, \xi_{2}\right)(m, n)\right), \quad \xi_{1}, \xi_{2}<\omega_{1}, \\
& \underline{\dot{h}}\left(\xi_{1}, \xi_{2}\right)=\bigcup_{n<\omega}\{n\} \times F^{-1}\left(h\left(\xi_{1}, \xi_{2}\right)(n)\right), \quad \xi_{1}, \xi_{2}<\omega_{1},
\end{aligned}
$$

then for each $\xi_{1}, \xi_{2}<\omega_{1}$,

$\Vdash_{P_{\omega_{2}}}$ " $\dot{G}\left(\xi_{1}, \xi_{2}\right)=\dot{G}\left(\xi_{1}, \xi_{2}\right)$ is the composition of $\xi_{1}$ and $\xi_{2}$ in $(\dot{G}, \dot{d})$ " and

$$
\begin{aligned}
& \Vdash_{P_{\omega_{2}}} \stackrel{\underline{h}}{\dot{h}}\left(\xi_{1}, \xi_{2}\right)=\dot{d}\left(\xi_{1}, \xi_{2}\right), \text { where } \underline{\dot{h}}\left(\xi_{1}, \xi_{2}\right) \text { is interpreted } \\
& \text { as explained at the start of the proof". }
\end{aligned}
$$

For all large enough $\alpha<\omega_{2}$, we have:

(i) $g: \omega_{1} \times \omega_{1} \rightarrow([\alpha] \leq \omega) \omega \times \omega$ and $h: \omega_{1} \times \omega_{1} \rightarrow([\alpha] \leq \omega)^{\omega}$.

(ii) $\dot{\dot{G}}\left(\xi_{1}, \xi_{2}\right)$ and $\underline{\dot{h}}\left(\xi_{1}, \xi_{2}\right)$ are $P_{\alpha}$-names for all $\xi_{1}, \xi_{2}<\omega_{1}$.

(iii) $\dot{f}$ is a $P_{\alpha}$-name.

Choose any such $\alpha$ of cofinality $\omega_{1}$. By (i) and (ii), the definitions of $\dot{G}\left(\xi_{1}, \xi_{2}\right)$ and $\underline{\dot{h}}\left(\xi_{1}, \xi_{2}\right)$ would not change if we used $g_{\alpha}$ instead of $g, h_{\alpha}$ instead of $h$, and $\bar{F}_{\alpha}$ instead of $F$. Then from the definitions of $\dot{G}_{\alpha}$ and $\dot{h}_{\alpha}$ we get

$\Vdash_{P_{\alpha}}$ " $\left(\dot{G}_{\alpha}, \dot{d}_{\alpha}\right)$ is a Baire metric group with left-invariant metric $\dot{d}_{\alpha}$ and $\dot{f}: \omega \rightarrow \omega_{1}$ has dense range in $(\dot{G}, \dot{d})$ ".

(Being a metric group is clearly absolute. That $\dot{f}$ is a function with dense range is also absolute. Being Baire is downward absolute.) Then $\dot{Q}_{\alpha}$ was chosen to add $\varphi$ and $V$ as required by the theorem.

[That the values of $\dot{G}_{\alpha}$ are well-orderings is absolute and then the ordertypes are equal in $V^{p}$ and $V^{P_{\alpha}}$. The fact that $\dot{G}_{\alpha} \cap \operatorname{ran} \varphi$ is a dense Baire subset of $\operatorname{ran} \varphi$ is preserved by the choice of $\bar{M}_{\alpha+1}^{\prime}$ and the fact that $P_{\alpha+1, \omega_{2}}$ is $\bar{M}_{\alpha+1}^{\prime \text {-cc.] }}$

This completes the proof of the theorem.

\section{References}

[Ba] J. E. Baumgartner, Iterated forcing, in: Surveys in Set Theory, A. R. D. Mathias (ed.), London Math. Soc. Lecture Note Ser. 87, Cambridge Univ. Press, Cambridge, 1983, 1-59. 
[Bu1993a] M. R. Burke, Liftings and the property of Baire in locally compact groups, Proc. Amer. Math. Soc. 117 (1993), 1075-1082.

[Bu1993b] - Liftings for Lebesgue measure, in: Set Theory of the Reals (Ramat Gan, 1991), Israel Math. Conf. Proc. 6, Bar-Ilan Univ., Ramat Gan, 1993, 119-150.

[BMMS] M. R. Burke, N. D. Macheras, K. Musiał and W. Strauss, Category product densities and liftings, Topology Appl. 153 (2006), 1164-1191.

[BM] M. R. Burke and A. W. Miller, Models in which every non-meager set is nonmeager in a nowhere dense Cantor set, Canad. J. Math. 57 (2005), 1139-1154.

[En] R. Engelking, General Topology, Heldermann, Berlin, 1989.

[Fr2003] D. H. Fremlin, Measure Theory, Vol. 4, Torres Fremlin, 2003.

[HR] E. Hewitt and K. A. Ross, Abstract Harmonic Analysis I, 2nd ed., Springer, Berlin, 1979.

[Hu] T. Husain, Introduction to Topological Groups, Saunders, Philadelphia, 1966.

[IT1967] A. Ionescu Tulcea and C. Ionescu Tulcea, On the existence of a lifting commuting with the left translations of an arbitrary locally compact group, Proc. Fifth Berkeley Sympos. Math. Statist. and Probability (Berkeley, CA, 1965/66), Vol. II: Contributions to Probability Theory, Part 1, Univ. of California Press, Berkeley, CA, 1967, 63-97.

[Jo] R. Johnson, Strong liftings which are not Borel liftings, Proc. Amer. Math. Soc. 80 (1980), 234-236.

[Kun] K. Kunen, Set Theory, North-Holland, Amsterdam, 1983.

[KP] J. Kupka and K. Prikry, Translation-invariant Borel liftings hardly ever exist, Indiana Univ. Math. J. 32 (1983), 717-731.

[Kur] K. Kuratowski, Topology, Vol. I, PWN, Warszawa, and Academic Press, New York, 1966.

[Lo] V. Losert, Some remarks on invariant liftings, in: Measure Theory Oberwolfach 1983, Lecture Notes in Math. 1089, Springer, Berlin, 1984, 95-110.

[LMZ] J. Lukeš, J. Malý and L. Zajíček, Fine Topology Methods in Real Analysis and Potential Theory, Lecture Notes in Math. 1189, Springer, 1986.

[Ma1958] D. Maharam, On a theorem of von Neumann, Proc. Amer. Math. Soc. 9 (1958), 987-994.

[Ma1977] —, Category, Boolean algebras and measure, in: General Topology and its Relations to Modern Analysis and Algebra, IV (Proc. Fourth Prague Topological Sympos., Prague, 1976), Part A, Lecture Notes in Math. 609, Springer, Berlin, 1977, 124-135.

[vN] J. von Neumann, Algebraische Repräsentanten der Funktionen „bis auf eine Menge vom Maße Null“, J. Reine Angew. Math. 165 (1931), 109-115.

[Re] J. P. Revalski, The Banach-Mazur game: history and recent developments, http://www.univ-ag.fr/aoc/activite/revalski/Banach-Mazur_Game.pdf.

[Sh1983] S. Shelah, Lifting problem of the measure algebra, Israel J. Math. 45 (1983), 90-96.

[Sh1998] - Proper and Improper Forcing, 2nd ed., Springer, Berlin, 1998.

[Ta] M. Talagrand, La pathologie des relèvements invariants, Proc. Amer. Math. Soc. 84 (1982), 379-382.

[To] S. Todorčević, Partition Problems in Topology, Contemp. Math. 84, Amer. Math. Soc., Providence, RI, 1989.

[TH-J] F. Topsøe and J. Hoffmann-Jørgensen, Analytic spaces and their application, in: Analytic Sets, C. A. Rogers et al. (eds.), Academic Press, London, 1980, $317-401$. 
Department of Mathematics and Statistics

University of Prince Edward Island

Charlottetown, PE, Canada C1A 4P3

E-mail: burke@upei.ca

Received 12 September 2005;

in revised form 13 December 2006 